\title{
Spiking-Free HGO-Based Dynamic Surface Control for Flexible Joint Manipulator with Unmeasurable States
}

\section{Dezhi Kong}

Northwestern Polytechnical University School of Mechanical Engineering https://orcid.org/0000-00020801-2730

\section{Wendong Wang ( $\nabla$ wdwang@nwpu.edu.cn )}

Shandong Jianzhu University https://orcid.org/0000-0002-2336-0477

\section{Yikai Shi}

Northwestern Polytechnical University

\section{Original Article}

Keywords: Flexible joint manipulator, Partial states feedback, High-gain observer, Mismatched uncertainties, Dynamic surface control, Spike suppression

Posted Date: December 15th, 2020

DOI: https://doi.org/10.21203/rs.3.rs-126111/v1

License: (c) (i) This work is licensed under a Creative Commons Attribution 4.0 International License. Read Full License 


\section{Title page}

\section{Spiking-free HGO-based Dynamic Surface Control for Flexible Joint Manipulator with Unmeasurable States}

De-Zhi Kong, received the B.S. degree in 2014 from Northeastern University, China. He received M.S. degree in 2017 from University of Sydney, Australia. He is currently a doctoral candidate of Northwestern Polytechnical University. His main research pursuit is robot control, nonlinear control theory .et.

Tel: +86-18629258478; E-mail: E-mail: dzkong@nwpu.edu.cn

Wen-Dong Wang, received the B.S degree in Mechanical Engineering and Automation from Shandong Jianzhu University, Jinan, in 2008 and the M.S and Ph.D. degree in Mechatronics from Northwestern Polytechnical University, Xi'an, China, in 2010 and 2014 , where he has been an associate Professor with the School of Mechanical Engineering since 2018. From 2011 to 2013, he was a research assistant in Robot and Automation Laboratory, University of Toronto, Canada. He has authored or co-authored more than 20 technical papers in international journals and conferences. His research interests include the design and control algorithm of rehabilitation and massage robot, human-robot interaction and application of artificial intelligence for healthcare robot.et.

E-mail: wdwang@nwpu.edu.cn

Yi-Kai Shi, is professor and doctoral supervisor of the School of Mechanical and Electrical Engineering of Northwestern Polytechnical University. Mainly engaged in the research of electronic mechanical engineering and electrical engineering .et.

E-mail: ykshi@nwpu.edu.cn

\section{Corresponding author: Wen-Dong Wang E-mail: wdwang@nwpu.edu.cn}




\title{
Spiking-free HGO-based Dynamic Surface Control for Flexible Joint Manipulator with Unmeasurable States
}

\author{
De-Zhi Kong ${ }^{1}$, • Wen-Dong Wang ${ }^{1} \cdot$ Yi-Kai Shi ${ }^{1}$
}

Received June xx, 201x; revised February xx, 201x; accepted March xx, 201x

(C) Chinese Mechanical Engineering Society and Springer-Verlag Berlin Heidelberg 2017

\begin{abstract}
For the flexible joint manipulator control system (FJMCS) with unmeasurable states, a novel partial states feedback control (PSFC) is proposed. Firstly, the unmeasurable states and the uncertainties are observed by a high-gain observer (HGO) simultaneously. Then, a dynamic surface controller is proposed based on the output of the HGO. The newly proposed controller has several advantages over existing methods. First, the proposed controller not only uses the estimate states to avoid using unmeasurable states, but also uses the estimation of uncertainties to enhance the robustness of FJMCS. Second, a novel spike suppression function (SSF) is developed to avoid the estimation spike problem in the existing HGO-based controllers. The closedloop system stability is proved by the Lyapunov theory. Simulation results demonstrate the effectiveness of the proposed controller.
\end{abstract}

Keywords: Flexible joint manipulator - Partial states feedback • High-gain observer $•$ Mismatched uncertainties $•$ Dynamic surface control $\bullet$ Spike suppression

\section{Introduction}

With the development of mechanical, control and sensor technology, the design of robot has made great progress and gradually replacing people in the fields of repetitive labor, emergency rescue and home service, etc. [1-4]. In robot system, the robot manipulator has a very important role as the end-effector of the robot which interacts with the external environment [5]. For the robot manipulator, many studies on control strategies for different kinds of robot manipulator components have been completed. And, among these strategies, the disturbance attenuation control takes an

Wen-Dong Wang

wdwang@nwpu.edu.cn important role since the robot manipulator system is a typical nonlinear uncertain system, and the uncertainties can be caused by the unmodeled dynamics, external environment and parameter uncertainties [6-10].

Compared with the conventional rigid robot manipulator (RRM), the flexible joint manipulator (FJM) has become a research hotspot increasingly since it has several advantages, such as smaller actuator, light weight, lower cost and better maneuverability, etc. [11-14]. However, the flexibility of FJM brings more complex uncertain characteristics of dynamic model. The dynamic model of conventional RRM is a low-order (two-order) matched uncertain system. But the dynamic model of FJM is a typical high-order (four-order) mismatched uncertain system.

To solve the mismatched uncertain problem, the backstepping control method (BCM) is a good candidate [15]. The main advantage of BCM is that it can suppress the mismatched uncertainties through a step-by-step recursive process [16]. Some BCM-based methods for FJM have been developed, such as BCM-based adaptive control [17], BCMbased fault-tolerant control [18], BCM-based neural network control [19], BCM-based intelligent control [20] and BCMbased L2-gain control [21]. For conventional BCM, the computations needed for back-stepping is very tedious and complicated, this is so called the problem of "differential explosion". To solve this problem, the dynamic surface method (DSM) was proposed [22-23]. The DSM not only can suppress the mismatched uncertainties by the step-bystep recursive process like BCM, but also adopt a low-pass filter to avoid the explosiveness of backstepping. Then an adaptive fault-tolerant control for FJM based on DSM was proposed [24], and the complex differential calculation for

1 School of Mechanical Engineering, Northwestern Polytechnical University, Xi'an 710072, P.R.China. 
backstepping in [17-21] can be avoid by the low-pass filter. However, in order to complete the step-by-step recursive process, the BCM-based and DSM-based controllers in [17$21,24]$ not only need to measure the states of link, but also need the states of motor for feedback. Thus, the measurement systems for the controllers in [17-21] are much more complex than the measurement system for the conventional RRM controller which only needs the states of link.

As a control method which only needs the measurements of partial states, the output feedback control (OFC) scheme can simplify the measurement system for high-order systems [25-28], significantly. Some control schemes for FJM based on OFC have been developed to simplify the measurement system. A control scheme which only requires the feedback of position states was developed for FJM [29], in which an observer was design to estimate the unmeasurable link and the actuator velocity, but the uncertainties of FJM were not considered in [29]. The unmeasurable velocities of link and motor were estimated by an approximate differential observer, and a complex adaptive control law was proposed to eliminate the effect of the uncertainties [30]. A high-gain observer was designed to generate pseudo signals for unmeasurable states, and an adaptive law of the projection type was developed to estimate the unknown feed-forward term [31].

To guarantee the observation and control precision, the observer gains of the observers in [29-31] should be chosen as the high gains. Thus, the observers used in [29-31] are typical high-gain observer (HGO). However, the undesirable spike problem can be caused by the high observer gains when the initial values of estimate and true states are not equal [32-33]. The spike problem may cause the saturation of control input and even lead to the instability of whole system. In addition, for the schemes in [30,31], on the basis of using observer, an additional robust control scheme should be adopted to eliminate the effect of uncertainties, thus the structures of controllers in [30,31] are very complex.

In this paper, a new partial states feedback control (PSFC) scheme based on DSM and HGO is proposed for the FJM. The main contributions of this paper are summarized as follows:

(1) The spike problem in the existing HGO-based schemes are solved: a novel spike suppression function (SSF) is adopted to solve the estimation spike problem in the existing HGO such as the observers in [29-31]. And, since the spike problem can be avoided even the observer gain is very high, a high observer gain can be adopted in the proposed scheme to guarantee the high control precision.

(2) Only partial states of FJM need to be measured: The proposed method only needs the same measurement states as conventional RRM by using the HGO to estimate the unknown states.

(3) The structure of proposed controller is simple: The unmeasurable states and uncertainties are estimated by HGO simultaneously. Then the uncertainties of FJM are compensated by using the estimation of uncertainties without requiring the additional robust control scheme such as the adaptive schemes used in [30,31]. Thus, the structure of proposed control scheme is simpler than the schemes in [30,31].

The remaining parts of this paper are as follows. In section 2 , the dynamic model, the design objective, and the motivation of this paper are expounded. The main results are presented in Section 3. In section 3.1, a high-gain observer (HGO) is developed. In section 3.2, a novel spike suppression function (SSF) is developed, and then a dynamic surface controller based on HGO and SSF is proposed. In section 4 , a simulation verifies the effectiveness of proposed HGO, SSF and controller. In section 5, the conclusion of the whole paper is presented.

\section{Problem Formulation}

\subsection{Dynamic Model of Flexible Joint Manipulator}

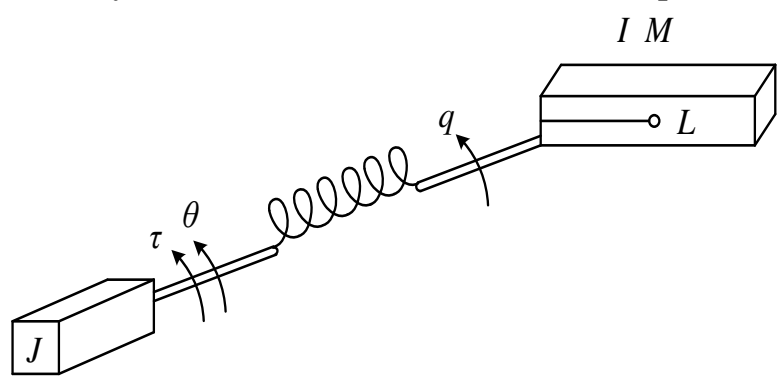

Figure 1 Structure of flexible manipulator

The flexible joint manipulator is shown schematically in Figure 1. The dynamic model of the flexible manipulator control system (FMCS) can be described as follows [34]:

$$
\left\{\begin{array}{l}
(I+\Delta I) \ddot{q}+(K+\Delta K)(q-\theta) \\
+(M g L+\Delta M g L) \sin (q)+d_{1}=0 \\
(J+\Delta J) \ddot{\theta}-(K+\Delta K)(q-\theta)+d_{2}=\tau
\end{array}\right.
$$

Where $q$ and $\dot{q}$ are the rotation angle and angular velocity of link, respectively. $\theta$ and $\dot{\theta}$ are the rotation angle and angular velocity of motor, respectively. $I$ and $J$ are the moment of inertia of link and motor, respectively. $M$ and $L$ represent the mass and the distance between the joint and the centroid of the link, respectively. $g$ is the 
gravity acceleration. $K$ represents the elastic stiffness of the flexible joint. $\tau$ is the motor torque. $\Delta I, \Delta K, \Delta J$ and $\triangle M g L$ represents the unmodeled dynamics of the FMCS. $d_{1}$ and $d_{2}$ denote the disturbance from the environment.

Define the lumped uncertainties $\bar{d}_{1}$ and $\bar{d}_{2}$ as

$$
\left\{\begin{array}{l}
\bar{d}_{1}=\Delta I \ddot{q}+\Delta K(q-\theta)+\Delta M g L \sin (q)+d_{1} \\
\bar{d}_{2}=\Delta J \ddot{\theta}-\Delta K(q-\theta)+d_{2}
\end{array}\right.
$$

Then, Equation (1) can be rewritten as

$$
\left\{\begin{array}{l}
\ddot{q}+\frac{K}{I}(q-\theta)+\frac{M g L}{I} \sin (q)+\frac{\bar{d}_{1}}{I}=0 \\
\ddot{\theta}-\frac{K}{J}(q-\theta)+\frac{1}{J} \bar{d}_{2}=\frac{1}{J} \tau
\end{array}\right.
$$

The states are defined as $x_{1}=q-q_{d}, x_{2}=\dot{q}-\dot{q}_{d}$, $x_{3}=K \theta / I, x_{4}=K \dot{\theta} / I$, and let the control input $u=\tau$, then the FJMCS can be rewritten as

$$
\left\{\begin{array}{l}
\dot{x}_{1}=x_{2} \\
\dot{x}_{2}=x_{3}+f_{1}\left(x_{1}\right)+\bar{\Delta}_{1} \\
\dot{x}_{3}=x_{4} \\
\dot{x}_{4}=b u+k_{1} x_{1}+k_{2} x_{3}+\bar{\Delta}_{2}
\end{array}\right.
$$

Where

$$
\left\{\begin{array}{l}
f_{1}\left(x_{1}\right)=-\frac{M g L}{I} \sin \left(x_{1}+q_{d}\right)-\frac{K\left(x_{1}+q_{d}\right)}{I}-\ddot{q}_{d} \\
k_{1}=\frac{K^{2}}{I J}, k_{2}=-\frac{K}{J} \\
b=\frac{K}{I J}, \bar{\Delta}_{1}=-\frac{\bar{d}_{1}}{I}, \bar{\Delta}_{2}=-\frac{K}{I J} \bar{d}_{2}
\end{array}\right.
$$

The control objective is to design the control input $u$ such that the tracking error $x_{1}$ approaches to zero, while ensuring that all closed loop signals are bounded.

\subsection{Problem Description and Purposes of this paper}

For the traditional control scheme for FJM in [17-21, 24], the link states $q, \dot{q}$ and the motor states $\theta, \dot{\theta}$ are both assumed to be measurable, but the RRM controllers only need the link states $q$ and $\dot{q}$. Thus, the measurement system for the controller in [17-21, 24] is much more complex than the measurement system for RRM. This motivates the first research topic of this paper, that is, designing a control scheme which only needs the same measurement states as RRM to simplify the measurement system of FJM. Thus, we have the following assumption:

Assumption 1: For the FJM system (1), $q$ and $\dot{q}$ are measurable, $\theta$ and $\dot{\theta}$ are unmeasurable.

To solve the unmeasurable problem, some schemes in [2628] have adopted an observer to estimate unmeasurable states. And a high observer gain should be chosen to guarantee the observation precision. However, an undesirable spike problem may be caused by the high observer gain (see [29-30]). To explain the spike problem, the high-gain observer in [28] can be give as following:

$$
\begin{gathered}
\dot{\hat{\boldsymbol{y}}}=\boldsymbol{A}_{y} \hat{\boldsymbol{y}}+\boldsymbol{k}\left(\hat{y}_{1}-y_{1}\right) \\
\hat{\boldsymbol{y}}=\left[\begin{array}{l}
\hat{y}_{1} \\
\hat{y}_{2}
\end{array}\right], \boldsymbol{A}_{y}=\left[\begin{array}{ll}
0 & 1 \\
0 & 0
\end{array}\right], \boldsymbol{k}=\left[\begin{array}{l}
-2 k_{0} \\
-k_{0}^{2}
\end{array}\right]
\end{gathered}
$$

where $y_{1}$ denotes the angle state, $\hat{y}_{1}$ and $\hat{y}_{2}$ are the estimate angle state and the estimate angular velocity state, respectively. $k_{0}$ is the observer gain.

Let estimate errors $\bar{y}_{1}=\hat{y}_{1}-y_{1}, \bar{y}_{2}=\hat{y}_{2}-\dot{y}_{1}$, we have

$$
\begin{gathered}
\overline{\boldsymbol{y}}=\boldsymbol{A}_{y} \overline{\boldsymbol{y}}+\boldsymbol{\delta} \\
\overline{\boldsymbol{y}}=\left[\begin{array}{l}
\bar{y}_{1} \\
\bar{y}_{2}
\end{array}\right], \quad \boldsymbol{A}_{y}=\left[\begin{array}{cc}
-2 k_{0} & 1 \\
-k_{0}^{2} & 0
\end{array}\right], \quad \boldsymbol{\delta}=\left[\begin{array}{l}
0 \\
\ddot{y}_{1}
\end{array}\right]
\end{gathered}
$$

The solutions for the differential equation (10) can be easily obtained as

$$
\overline{\boldsymbol{y}}(t)=e^{\boldsymbol{A}_{y} t} \overline{\boldsymbol{y}}(0)+\int_{t_{0}}^{t} e^{\boldsymbol{A}_{y}(t-v)} \ddot{y_{1}} d v
$$

where $e$ is the e constant. $t$ is the time and the initial time is 0 in this paper. Since the eigenvalues of $A_{y}$ both are $\lambda\left(A_{y}\right)=-k_{0}$, for the small time $t=1 / k_{0}, e^{\boldsymbol{A}_{y} t} \overline{\boldsymbol{y}}(0)$ can be rewritten as

$$
e^{\boldsymbol{A}_{\mathrm{y}} / k_{0}} \overline{\boldsymbol{y}}(0)=\left[\begin{array}{c}
e^{-1} \bar{y}_{2}(0) / k_{0} \\
-k_{0} e^{-1} \bar{y}_{1}(0)+2 e^{-1} \bar{y}_{2}(0)
\end{array}\right]
$$

It can be known that $k_{0} e^{-1} \bar{y}_{1}(0)$ is a very large value if $k_{0}$ is a high gain and the initial estimate state error $\bar{y}_{1}(0)=\hat{y}_{1}-y_{1} \neq 0$ is not equal to 0 . The large values $k_{0} e^{-1} \bar{y}_{1}(0)$ may lead to the saturation of control input and even the instability of system, this is the undesirable spike problem. Actually, the initial values of true state $y_{1}(0)$ 
cannot be known in advance for the most of cases. Thus, the initial estimate state error $\bar{y}_{1}(0)$ maybe not equal to 0 and even a large value. If the initial value of true states $y_{1}(0)$ is unknown, to avoid the potential spike problem, the HGObased controllers in [29-31] cannot adopt the enough high observer gain to guarantee the control precision. This motivates the second research topic of this paper, that is, design a new scheme not only can guarantee high precision, but also eliminate the undesirable spike problem in any cases

On the other hand, from Equation (4), it is clear that FJMCS is a typical mismatched uncertain system. To suppress the matched and mismatched uncertainties, the conventional control schemes in [30,31] for FJMCS need an additional robust scheme. Thus, the structures of the controllers in [30,31] are very complex. This motivates the third research topic of this paper, that is, avoiding using additional robust scheme. The motivation of this paper is summarized as follows:

Motivation of This Paper: (1) To avoid using the unmeasurable states, the unknown states will be estimated by designing a HGO in following section 3.1. (2) To use the high observer gain, while avoiding the spike problem, a novel SSF will be proposed in the following section 3.2. (3) To suppress the uncertainties without using additional complex method, the proposed HGO are used to estimate not only the unmeasurable state but also the uncertainties.

\section{Main Result}

\subsection{High-gain Observer}

For the system (13), since $\theta$ and $\dot{\theta}$ are unmeasurable, the states $x_{3}$ and $x_{4}$ are unknown. Combining unknown states with uncertainties to construct the new states. The new states are defined as $p_{1}=x_{1}, p_{2}=x_{2}, p_{3}=x_{3}+\bar{\Delta}_{1}$, $p_{4}=x_{4}+\dot{\bar{\Delta}}_{1}$ and the uncertainty $d_{f}=\bar{\Delta}_{2}+\ddot{\bar{\Delta}}_{1}-k_{2} \bar{\Delta}_{1}$. Then the FJMCS (14) can be rewritten as

$$
\left\{\begin{array}{l}
\dot{p}_{1}=p_{2} \\
\dot{p}_{2}=p_{3}+f_{1}\left(p_{1}\right) \\
\dot{p}_{3}=p_{4} \\
\dot{p}_{4}=b u+k_{1} p_{1}+k_{2} p_{3}+d_{f}
\end{array}\right.
$$

Since $p_{1}=x_{1}$, the control objective can be equivalent to $p_{1} \rightarrow 0$.

If the states $p_{3}$ and $p_{4}$ are known, the system (12) is a matched uncertain system. Let the matched uncertainty $d_{f}=p_{5}$, where $p_{5}$ is the extended state. The system (12) can be extended as

$$
\dot{\boldsymbol{p}}=\boldsymbol{A}_{p 1} \boldsymbol{p}+\boldsymbol{A}_{p 2} \boldsymbol{p}+\boldsymbol{B} u+\boldsymbol{f}_{p}+\boldsymbol{d}
$$

where

$$
\begin{aligned}
\boldsymbol{A}_{p 1}= & {\left[\begin{array}{lllll}
0 & 1 & 0 & 0 & 0 \\
0 & 0 & 1 & 0 & 0 \\
0 & 0 & 0 & 1 & 0 \\
0 & 0 & 0 & 0 & 1 \\
0 & 0 & 0 & 0 & 0
\end{array}\right], \boldsymbol{A}_{p 2}=\left[\begin{array}{ccccc}
0 & 0 & 0 & 0 & 0 \\
0 & 0 & 0 & 0 & 0 \\
0 & 0 & 0 & 0 & 0 \\
k_{1} & 0 & k_{2} & 0 & 0 \\
0 & 0 & 0 & 0 & 0
\end{array}\right] } \\
& \boldsymbol{p}=\left[\begin{array}{c}
p_{1} \\
p_{2} \\
p_{3} \\
p_{4} \\
p_{5}
\end{array}\right], \boldsymbol{f}_{p}=\left[\begin{array}{c}
0 \\
f_{1}\left(p_{1}\right) \\
0 \\
0 \\
0
\end{array}\right], \boldsymbol{B}=\left[\begin{array}{l}
0 \\
0 \\
0 \\
b \\
0
\end{array}\right], \boldsymbol{d}=\left[\begin{array}{c}
0 \\
0 \\
0 \\
0 \\
\dot{d}_{f}
\end{array}\right]
\end{aligned}
$$

The following assumption is needed:

Assumption 2: The uncertainty is bounded and satisfies $\left\|d_{f}\right\| \leq d_{\max }$ and $\left\|\dot{d}_{f}\right\| \leq \bar{d}_{\max }$, where $d_{\max }$ and $\bar{d}_{\max }$ are positive constants.

To satisfy the Assumption 1, we can know that the lumped uncertainties $\bar{d}_{1}$ and $\bar{d}_{\underline{2}}$ in (1) should satisfy the condition: $\bar{d}_{1}^{(j)}$ and $\bar{d}_{1}^{(i)}$ are bounded, where $j=0,1,2,3$ and $i=0,1$.

Then a five-order HGO is proposed in the following theorem to estimate unknown states $p_{3}, p_{4}$ and uncertainty $d_{f}$, simultaneously.

Theorem 1: For the system (12), a HESO is constructed as

$$
\dot{\hat{\boldsymbol{p}}}=\boldsymbol{A}_{p 1} \hat{\boldsymbol{p}}+\boldsymbol{A}_{p 2} \hat{\boldsymbol{p}}+\boldsymbol{B} u+\boldsymbol{f}_{p}+\boldsymbol{C}\left(p_{1}-\hat{p}_{1}\right)
$$

where

$$
\begin{gathered}
\hat{\boldsymbol{p}}=\left[\begin{array}{lllll}
\hat{p}_{1} & \hat{p}_{2} & \hat{p}_{3} & \hat{p}_{4} & \hat{p}_{5}
\end{array}\right]^{T} \\
\boldsymbol{C}=\left[\begin{array}{lllll}
5 c_{0} & 10 c_{0}^{2} & 10 c_{0}^{3} & 5 c_{0}^{4} & c_{0}^{5}
\end{array}\right]^{T}
\end{gathered}
$$

If assumption 1 and 2 are valid and the observer gain satisfies $c_{0}>\bar{k}$, where $\bar{k}=\left\|\boldsymbol{A}_{p 2}^{T}+\boldsymbol{A}_{p 2}\right\| / 2$, then the estimate error $\overline{\boldsymbol{p}}=\boldsymbol{p}-\hat{\boldsymbol{p}}$ will converge to following small region:

$$
\|\overline{\boldsymbol{p}}\| \leq \frac{\bar{d}_{\max }}{\left(c_{0}-\bar{k}\right)} \text {, if } t \rightarrow \infty
$$


Proof (Proof of Theorem 1): Define the estimate error vector as

$$
\begin{gathered}
\overline{\boldsymbol{p}}=\boldsymbol{p}-\hat{\boldsymbol{p}} \\
\overline{\boldsymbol{p}}=\left[\begin{array}{lllll}
\bar{p}_{1} & \bar{p}_{2} & \bar{p}_{3} & \bar{p}_{4} & \bar{p}_{5}
\end{array}\right]^{T}
\end{gathered}
$$

Differentiating $\overline{\boldsymbol{p}}$ gives

$$
\dot{\bar{p}}=\dot{\boldsymbol{p}}-\dot{\hat{\boldsymbol{p}}}
$$

Substituting (13) and (13) into (22) yields

$$
\begin{aligned}
\dot{\overline{\boldsymbol{p}}} & =\boldsymbol{A}_{p 1} \boldsymbol{p}+\boldsymbol{A}_{p 2} \boldsymbol{p}+\boldsymbol{B} u+\boldsymbol{f}_{p}+\boldsymbol{d}-\boldsymbol{A}_{p 1} \hat{\boldsymbol{p}} \\
& -\boldsymbol{A}_{p 2} \hat{\boldsymbol{p}}-\boldsymbol{B} u-\boldsymbol{f}_{p}-\boldsymbol{C}\left(p_{1}-\hat{p}_{1}\right) \\
& =\boldsymbol{A}_{p 1}(\boldsymbol{p}-\hat{\boldsymbol{p}})-\boldsymbol{C}\left(p_{1}-\hat{p}_{1}\right)+\boldsymbol{A}_{p 2}(\boldsymbol{p}-\hat{\boldsymbol{p}})+\boldsymbol{d} \\
& =\boldsymbol{A} \overline{\boldsymbol{p}}+\boldsymbol{A}_{p 2} \overline{\boldsymbol{p}}+\boldsymbol{d}
\end{aligned}
$$

where

$$
\boldsymbol{A}=\left[\begin{array}{ccccc}
-5 c_{0} & 1 & 0 & 0 & 0 \\
-10 c_{0}^{2} & 0 & 1 & 0 & 0 \\
-10 c_{0}^{3} & 0 & 0 & 1 & 0 \\
-5 c_{0}^{4} & 0 & 0 & 0 & 1 \\
-c_{0}^{5} & 0 & 0 & 0 & 0
\end{array}\right]
$$

Construct Lyapunov function $V_{1}$ as

$$
V_{1}=\frac{1}{2} \overline{\boldsymbol{p}}^{T} \overline{\boldsymbol{p}}
$$

Then calculating the time derivative of $V_{1}$ along the trajectories of Equation (23), we have

$$
\begin{aligned}
\dot{V}_{1} & =\frac{1}{2}\left(\dot{\bar{p}}^{T} \overline{\boldsymbol{p}}+\overline{\boldsymbol{p}}^{T} \dot{\overline{\boldsymbol{p}}}\right) \\
& =\frac{1}{2}\left(\left(A \overline{\boldsymbol{p}}+\boldsymbol{A}_{p 2} \overline{\boldsymbol{p}}+\boldsymbol{d}\right)^{T} \overline{\boldsymbol{p}}+\overline{\boldsymbol{p}}^{T}\left(\boldsymbol{A} \overline{\boldsymbol{p}}+\boldsymbol{A}_{p 2} \overline{\boldsymbol{p}}+\boldsymbol{d}\right)\right) \\
& =\frac{1}{2}\left(\overline{\boldsymbol{p}}^{T} \boldsymbol{A}^{T} \overline{\boldsymbol{p}}+\overline{\boldsymbol{p}}^{T} \boldsymbol{A}_{p 2}^{T} \overline{\boldsymbol{p}}+\boldsymbol{d}^{T} \overline{\boldsymbol{p}}+\overline{\boldsymbol{p}}^{T} \boldsymbol{A} \overline{\boldsymbol{p}}+\overline{\boldsymbol{p}}^{T} \boldsymbol{A}_{p 2} \overline{\boldsymbol{p}}+\overline{\boldsymbol{p}}^{T} \boldsymbol{d}\right) \\
& =\frac{1}{2}\left(\overline{\boldsymbol{p}}^{T}\left(\boldsymbol{A}^{T}+\boldsymbol{A}\right) \overline{\boldsymbol{p}}+\overline{\boldsymbol{p}}^{T}\left(\boldsymbol{A}_{p 2}^{T}+\boldsymbol{A}_{p 2}\right) \overline{\boldsymbol{p}}+\boldsymbol{d}^{T} \overline{\boldsymbol{p}}+\overline{\boldsymbol{p}}^{T} \boldsymbol{d}\right)
\end{aligned}
$$

Since the largest eigenvalue $\lambda_{\max }(\boldsymbol{A})=\lambda_{\max }\left(\boldsymbol{A}^{T}\right)=-c_{0}$, then Equation (26) can be rewritten as

$$
\dot{V}_{1} \leq-c_{0} \overline{\boldsymbol{p}}^{T} \overline{\boldsymbol{p}}+\left\|\boldsymbol{A}_{p 2}^{T}+\boldsymbol{A}_{p 2}\right\| \overline{\boldsymbol{p}}^{T} \overline{\boldsymbol{p}}+\|\boldsymbol{d}\|\|\overline{\boldsymbol{p}}\|
$$

Since $k_{1}$ and $k_{2}$ are known system parameters, $\left\|\boldsymbol{A}_{p 2}^{T}+\boldsymbol{A}_{p 2}\right\| / 2$ is known. Let $\bar{k}=\left\|\boldsymbol{A}_{p 2}^{T}+\boldsymbol{A}_{p 2}\right\| / 2$, we have

$$
\dot{V}_{1} \leq-c_{0} \overline{\boldsymbol{p}}^{T} \overline{\boldsymbol{p}}+\overline{k \overline{\boldsymbol{p}}}^{T} \overline{\boldsymbol{p}}+\|\boldsymbol{d}\|\|\overline{\boldsymbol{p}}\|
$$

Since Assumption 2 is valid, Equation (26) can be rewritten as

$$
\begin{aligned}
\dot{V}_{1} & \leq-c_{0} \overline{\boldsymbol{p}}^{T} \overline{\boldsymbol{p}}+\bar{k}^{T} \overline{\boldsymbol{p}} \overline{\boldsymbol{p}}+\bar{d}_{\text {max }}\|\overline{\boldsymbol{p}}\| \\
& =-\left(\left(c_{0}-\bar{k}\right)\|\overline{\boldsymbol{p}}\|-\bar{d}_{\text {max }}\right)\|\overline{\boldsymbol{p}}\|
\end{aligned}
$$

Let $c_{0}-\bar{k}>0$, then $\|\overline{\boldsymbol{p}}\|$ is always bounded as

$$
\|\overline{\boldsymbol{p}}\| \leq p_{g \max }, \text { if } t>0
$$

where $p_{g \text { max }}$ is a unknown positive constant.

From Equation (29), it can be known that $\dot{V}_{1}<0$ if $\|\overline{\boldsymbol{p}}\|>\bar{d}_{\max } /\left(c_{0}-\bar{k}\right)$ and $\|\overline{\boldsymbol{p}}\| \neq 0$, thus $\|\overline{\boldsymbol{p}}\|$ will converge to following region:

$$
\|\overline{\boldsymbol{p}}\| \leq \frac{\bar{d}_{\max }}{\left(c_{0}-\bar{k}\right)}, \text { if } t \rightarrow \infty
$$

Remark 3.1: From Equation (31), it can be known that the observer gain $c_{0}$ should be chosen large enough to ensure the estimation error is small enough. Thus, the proposed observer (20) also is a typical HGO. The undesirable spike problem may be caused by the high observer gain as mentioned above in section 1.2. To solve this potential problem, a novel spike suppression function (SSF) will be proposed in the following control design.

\subsection{Arm Dynamic Surface Controller Based on HGO and SSF}

Based on the estimate states $\hat{p}_{3}$ and $\hat{p}_{4}$, and the estimation of uncertainty $\hat{p}_{5}$, a DSM control scheme for the FJMCS (12) will be proposed by following four backstepping steps. And a novel SSF is adopted in the proposed controller to deal with the aforementioned spike problem of HGO.

Step 1: Define a tracking error as $s_{1}=p_{1}$, the virtual control of $p_{2}$ is designed as

$$
p_{2 d}=-\varepsilon_{1} s_{1}
$$

Define a low-pass filter as

$$
\tau_{2} \dot{p}_{2 c}+p_{2 c}=p_{2 d}
$$


where $\varepsilon_{1}$ and $\tau_{2}$ are positive constant.

Step 2: Define $s_{2}=p_{2}-p_{2 c}$, the virtual control of $p_{3}$ is taken as

$$
p_{3 d}=-\varepsilon_{2} s_{2}-f_{1}\left(p_{1}\right)+\dot{p}_{2 c}
$$

Define a low-pass filter as

$$
\tau_{3} \dot{p}_{3 c}+p_{3 c}=p_{3 d}
$$

where $\varepsilon_{2}$ and $\tau_{3}$ are positive constant.

Step 3: Define $s_{3}=p_{3}-p_{3 c}$, the virtual control of $p_{4}$ is taken as

$$
p_{4 d}=-\varepsilon_{3}\left(\hat{p}_{3}-p_{3 c}\right)+\dot{p}_{3 c}
$$

Define a low-pass filter as

$$
\tau_{4} \dot{p}_{4 c}+p_{4 c}=p_{4 d}
$$

where $\varepsilon_{3}$ and $\tau_{4}$ are positive constant.

Step4: Define $s_{4}=p_{4}-p_{4 c}$, the controller is designed as

$$
\begin{aligned}
u= & b^{-1}\left[-\varepsilon_{4} \chi_{1}(t)\left(\hat{p}_{4}-p_{4 c}\right)-k_{1}\left(p_{1}+q_{d}\right)-k_{2} \chi_{2}(t) \hat{p}_{3}\right. \\
& \left.-k_{2}\left(1-\chi_{2}(t)\right) p_{4 c}-\chi_{3}(t) \hat{p}_{5}+\chi_{4}(t) \dot{p}_{4 c}\right]
\end{aligned}
$$

where $\chi_{i}(t)(i=1,2,3,4)$ is the spike suppression function (SSF). The SSF $\chi_{i}(t)$ is defined as

$$
\chi_{i}(t)=\left(1-\kappa_{i} e^{-\eta_{i} t}\right)^{\psi_{i}}
$$

The constant parameters are chosen as $0<\kappa_{i}<1, \eta_{i}>0$ and $\psi_{i}>1$. Then, for $i=1,2,3,4$, it can be easily obtained that

$$
\begin{gathered}
\dot{\chi}_{i}(t)=\psi_{i}\left(1-\kappa_{i} e^{-\eta_{i} t}\right)^{\psi_{i}-1} \kappa_{i} \eta_{i} e^{-\eta_{i} t}>0 \\
0 \leq \chi_{i}(t) \leq 1
\end{gathered}
$$

Remark 3.2: As mentioned in section 2.2, the spike problem of HGO can be caused by a high observer gain $c_{0}$ if $p_{1}(0) \neq \hat{p}_{1}(0)$ in the initial small time period. From Equation (38), it is clear that the spike values of HGO can be taken into the control input by $\hat{p}_{4}, p_{4 c}, \hat{p}_{3}, \hat{p}_{5}, \dot{p}_{4 c}$ which are contain the outputs of HGO directly or indirectly. Thus, for the proposed controller (38), $\chi_{1}(t), \chi_{2}(t), \chi_{3}(t)$ and $\chi_{4}(t)$ are adopted to suppress the spike values of $\hat{p}_{4}-p_{4 c}, \hat{p}_{3}, \hat{p}_{5}$ and $\dot{p}_{4 c}$. From Equation (39), it can be known that $\chi_{i}(t)$ is small enough in the initial small time period by chosen the parameters reasonably, the spike values taken by $\hat{p}_{4}, p_{4 c}, \hat{p}_{3}, \hat{p}_{5}, \dot{p}_{4 c}$ can be suppressed by the proposed SSF.

Remark 3.3: Since $\chi_{i}(t) \rightarrow 1$ if $t \rightarrow \infty$, the stability and control precision of the proposed controller will not be affected by the SSF. The detail stability and control precision analysis of the proposed controller with SSF will be given in the Theorem 2.

Before giving the Theorem 2, the following assumption which is commonly used in DSM (such as in the section 3.2 in [22], the author assumed that the derivative of virtual control input is bounded as a positive constant) should be assumed to be valid throughout this paper:

Assumption 3: The vector $\boldsymbol{p}_{f}=\left[\begin{array}{lll}\dot{p}_{2 d} & \dot{p}_{3 d} & \dot{p}_{4 d}\end{array}\right]^{T}$ is bounded, and satisfies $\left\|\boldsymbol{p}_{f}\right\| \leq p_{f \max }$, where $p_{f \max }$ is a unknown positive constant.

Theorem 2: Consider the FMCS (12) with the controller (38), if assumption 1, 2 and 3 are valid, and the parameters are set reasonably, then the convergence of the tracking error $s_{j}(j=1,2,3,4)$ to a small neighborhood of zero can be guaranteed.

Proof (Proof of Theorem 2): Define the errors of low-pass filter as

$$
\left\{\begin{array}{l}
e_{2}=p_{2 c}-p_{2 d} \\
e_{3}=p_{3 c}-p_{3 d} \\
e_{4}=p_{4 c}-p_{4 d}
\end{array}\right.
$$

From Equations (33), (35)and (37), we have

$$
\left\{\begin{array}{l}
\dot{e}_{2}=-\tau_{2}^{-1} e_{2}-\dot{p}_{2 d} \\
\dot{e}_{3}=-\tau_{3}^{-1} e_{3}-\dot{p}_{3 d} \\
\dot{e}_{4}=-\tau_{4}^{-1} e_{4}-\dot{p}_{4 d}
\end{array}\right.
$$

Then calculating the time derivative of $s_{j}(j=1,2,3,4)$ along the trajectories of Equation (12), we get

$$
\left\{\begin{array}{l}
\dot{s}_{1}=p_{2}+p_{2 d}-p_{2 d} \\
\dot{s}_{2}=p_{3}+f_{1}\left(p_{1}\right)-\dot{p}_{2 c}+p_{3 d}-p_{3 d} \\
\dot{s}_{3}=p_{4}-\dot{p}_{3 c}+p_{4 d}-p_{4 d} \\
\dot{s}_{4}=b u+k_{1} p_{1}+k_{2} p_{3}+d_{f}-\dot{p}_{4 c}
\end{array}\right.
$$

Substituting Equations (32), (34), (36)and (38) into (44), we have 


$$
\left\{\begin{aligned}
\dot{s}_{1}= & -\varepsilon_{1} s_{1}+p_{2}-p_{2 d} \\
\dot{s}_{2}= & -\varepsilon_{2} s_{2}+p_{3}-p_{3 d} \\
\dot{s}_{3}= & -\varepsilon_{3}\left(\hat{p}_{3}-p_{3 c}\right)+p_{4}-p_{4 d} \\
\dot{s}_{4}= & -\varepsilon_{4} \chi_{1}(t)\left(\hat{p}_{4}-p_{4 c}\right)-k_{2} \chi_{2}(t) \hat{p}_{3}-k_{2}\left(1-\chi_{2}(t)\right) p_{3 c} \\
& -\chi_{3}(t) \hat{p}_{5}+\chi_{4}(t) \dot{p}_{4 c}+k_{2} p_{3}+d_{f}-\dot{p}_{4 c}
\end{aligned}\right.
$$

Then Equation (45) can be rewritten as

$$
\left\{\begin{aligned}
\dot{s}_{1}= & -\varepsilon_{1} s_{1}+p_{2}-p_{2 c}+p_{2 c}-p_{2 d} \\
\dot{s}_{2}= & -\varepsilon_{2} s_{2}+p_{3}-p_{3 c}+p_{3 c}-p_{3 d} \\
\dot{s}_{3}= & -\varepsilon_{3}\left(p_{3}-p_{3 c}-\left(p_{3}-\hat{p}_{3}\right)\right)+p_{4}-p_{4 c}+p_{4 c}-p_{4 d} \\
\dot{s}_{4}= & -\varepsilon_{4} \chi_{1}(t)\left(\left(p_{4}-p_{4 c}\right)-\left(p_{4}-\hat{p}_{4}\right)\right)+k_{2} \chi_{2}(t) \\
& \left(p_{3}-\hat{p}_{3}\right)+k_{2}\left(1-\chi_{2}(t)\right)\left(p_{3}-p_{3 c}\right)+\chi_{3}(t) \\
& \left(d_{f}-\hat{p}_{5}\right)-\left(1-\chi_{4}(t)\right) \dot{p}_{4 c}+\left(1-\chi_{3}(t)\right) d_{f}
\end{aligned}\right.
$$

Combining Equations (46) with (42) yields

$$
\left\{\begin{aligned}
\dot{s}_{1}= & -\varepsilon_{1} s_{1}+s_{2}+e_{2} \\
\dot{s}_{2}= & -\varepsilon_{2} s_{2}+s_{3}+e_{3} \\
\dot{s}_{3}= & -\varepsilon_{3} s_{3}+\varepsilon_{3} \bar{p}_{3}+s_{4}+e_{4} \\
\dot{s}_{4}= & -\varepsilon_{4} \chi_{1}(t) s_{4}+\varepsilon_{4} \chi_{1}(t) \bar{p}_{4}+k_{2} \chi_{2}(t) \bar{p}_{3}+ \\
& k_{2}\left(1-\chi_{2}(t)\right) s_{3}+\chi_{3}(t) \bar{p}_{5}-\left(1-\chi_{4}(t)\right) \dot{p}_{4 c} \\
& +\left(1-\chi_{3}(t)\right) d_{f}
\end{aligned}\right.
$$

Construct Lyapunov function $V_{2}$ as

$$
V_{2}=\frac{1}{2} \boldsymbol{s}^{T} \boldsymbol{s}+\frac{1}{2} \boldsymbol{e}^{T} \boldsymbol{e}
$$

where

$$
\begin{gathered}
\boldsymbol{s}=\left[\begin{array}{llll}
s_{1} & s_{2} & s_{3} & s_{4}
\end{array}\right]^{T} \\
\boldsymbol{e}=\left[\begin{array}{lll}
e_{2} & e_{3} & e_{4}
\end{array}\right]^{T}
\end{gathered}
$$

Calculating the time derivative of Equation (48) along the trajectories of Equations (43) and (47) yields

$$
\begin{aligned}
\dot{V}_{2}= & \frac{1}{2}\left(\dot{\boldsymbol{s}}^{T} \boldsymbol{s}+\boldsymbol{s}^{T} \dot{\boldsymbol{s}}\right)+\frac{1}{2}\left(\dot{\boldsymbol{e}}^{T} \boldsymbol{e}+\boldsymbol{e}^{T} \dot{\boldsymbol{e}}\right) \\
= & \left(\dot{s}_{1} s_{1}+\dot{s}_{2} s_{2}+\dot{s}_{3} s_{3}+\dot{s}_{4} s_{4}\right)+\left(\dot{e}_{2} e_{2}+\dot{e}_{3} e_{3}+\dot{e}_{4} e_{4}\right) \\
= & -\varepsilon_{1} s_{1}^{2}+s_{1} s_{2}+s_{1} e_{2}-\varepsilon_{2} s_{2}^{2}+s_{2} s_{3}+s_{2} e_{3}-\varepsilon_{3} s_{3}^{2} \\
& +\varepsilon_{3} s_{3} \bar{p}_{3}+s_{3} s_{4}+s_{3} e_{4}-\varepsilon_{4} \chi_{1}(t) s_{4}^{2}+\varepsilon_{4} \chi_{1}(t) s_{4} \bar{p}_{4} \\
& +k_{2} \chi_{2}(t) s_{4} \bar{p}_{3}+\chi_{3}(t) s_{4} \bar{p}_{5}-\tau_{2}^{-1} e_{2}^{2}-\dot{p}_{2 d} e_{2}-\tau_{3}^{-1} e_{3}^{2} \\
& -\dot{p}_{3 d} e_{3}-\tau_{4}^{-1} e_{4}^{2}-\dot{p}_{4 d} e_{4}+k_{2}\left(1-\chi_{2}(t)\right) s_{3} s_{4}+ \\
& \left(1-\chi_{3}(t)\right) d_{f} s_{4}-\left(1-\chi_{4}(t)\right) \dot{p}_{4 c} s_{4}
\end{aligned}
$$

Using Young's inequality [22-24], obtains

$$
\begin{aligned}
\dot{V}_{2} \leq & -\varepsilon_{1} s_{1}^{2}+\left(s_{1}^{2}+s_{2}^{2}\right) / 2+\left(s_{1}^{2}+e_{2}^{2}\right) / 2-\varepsilon_{2} s_{2}^{2}+\left(s_{2}^{2}+s_{3}^{2}\right) / 2+ \\
& \left(s_{2}^{2}+e_{3}^{2}\right) / 2-\varepsilon_{3} s_{3}^{2}+\varepsilon_{3}\left(s_{3}^{2}+\bar{p}_{3}^{2}\right) / 2+\left(s_{3}^{2}+s_{4}^{2}\right) / 2+\left(s_{3}^{2}+e_{4}^{2}\right) / 2 \\
& -\varepsilon_{4} \chi_{1}(t) s_{4}^{2}+\varepsilon_{4} \chi_{1}(t)\left(s_{4}^{2}+\bar{p}_{4}^{2}\right) / 2+k_{2} \chi_{2}(t)\left(s_{4}^{2}+\bar{p}_{3}^{2}\right) / 2+\chi_{3}(t) \\
& \left(s_{4}^{2}+\bar{p}_{5}^{2}\right) / 2-\tau_{2}^{-1} e_{2}^{2}+\left(\dot{p}_{2 d}^{2}+e_{2}^{2}\right) / 2-\tau_{3}^{-1} e_{3}^{2}+\left(\dot{p}_{3 d}^{2}+e_{3}^{2}\right) / 2-\tau_{4}^{-1} e_{4}^{2} \\
& +\left(\dot{p}_{4 d}^{2}+e_{4}^{2}\right) / 2+k_{2}\left(1-\chi_{2}(t)\right)\left(s_{3}^{2}+s_{4}^{2}\right) / 2+\left(1-\chi_{3}(t)\right)\left(d_{f}^{2}+s_{4}^{2}\right) / 2 \\
& +\left(1-\chi_{4}(t)\right)\left(\dot{p}_{4 c}^{2}+s_{4}^{2}\right) / 2 \\
= & -\left(\varepsilon_{1}-1\right) s_{1}^{2}-\left(\varepsilon_{2}-3 / 2\right) s_{2}^{2}-\left(\varepsilon_{3} / 2-3 / 2-k_{2}\left(1-\chi_{2}(t)\right) / 2\right) s_{3}^{2} \\
& -\left(\varepsilon_{4} \chi_{1}(t) / 2-3 / 2+\chi_{4}(t) / 2\right) s_{4}^{2}-\left(\tau_{2}^{-1}-1\right) e_{2}^{2}-\left(\tau_{3}^{-1}-1\right) e_{3}^{2}- \\
& \left(\tau_{4}^{-1}-1\right) e_{4}^{2}+\left(\varepsilon_{3} / 2+k_{2} \chi_{2}(t) / 2\right) \bar{p}_{3}^{2}+\varepsilon_{4} \chi_{1}(t) \bar{p}_{4}^{2} / 2+\chi_{3}(t) \bar{p}_{5}^{2} / 2+ \\
& \dot{p}_{2 d}^{2} / 2+\dot{p}_{3 d}^{2} / 2+\dot{p}_{4 d}^{2} / 2+\left(1-\chi_{3}(t)\right) d_{f}^{2} / 2+\left(1-\chi_{4}(t)\right) \dot{p}_{4 c}^{2} / 2
\end{aligned}
$$

Define the functions $f_{3}(t)$ and $f_{4}(t)$ as

$$
\begin{aligned}
f_{3}(t) & =\varepsilon_{3} / 2-3 / 2-k_{2}\left(1-\chi_{2}(t)\right) / 2 \\
& =\varepsilon_{3} / 2-3 / 2-k_{2}\left(1-\left(1-\kappa_{2} e^{-\eta_{2} t}\right)^{\psi_{2}}\right) / 2
\end{aligned}
$$

$$
\begin{aligned}
f_{4}(t) & =\varepsilon_{4} \chi_{1}(t) / 2-3 / 2+\chi_{4}(t) / 2 \\
& =\varepsilon_{4}\left(1-\kappa_{1} e^{-\eta_{1} t}\right)^{\psi_{1}} / 2-3 / 2+\left(1-\kappa_{4} e^{-\eta_{4} t}\right)^{\psi_{4}} / 2
\end{aligned}
$$

Then we have

$$
\begin{aligned}
\dot{V}_{2} \leq & -\left(\varepsilon_{1}-1\right) s_{1}^{2}-\left(\varepsilon_{2}-3 / 2\right) s_{2}^{2}-f_{3}(t) s_{3}^{2}-f_{4}(t) s_{4}^{2} \\
& -\left(\tau_{2}^{-1}-1\right) e_{2}^{2}-\left(\tau_{3}^{-1}-1\right) e_{3}^{2}-\left(\tau_{4}^{-1}-1\right) e_{4}^{2}+ \\
& \left(\varepsilon_{3} / 2+k_{2} \chi_{2}(t) / 2\right) \bar{p}_{3}^{2}+\varepsilon_{4} \chi_{1}(t) \bar{p}_{4}^{2} / 2+ \\
& \chi_{3}(t) \bar{p}_{5}^{2} / 2+\dot{p}_{2 d}^{2} / 2+\dot{p}_{3 d}^{2} / 2+\dot{p}_{4 d}^{2} / 2+ \\
& \left(1-\chi_{3}(t)\right) d_{f}^{2} / 2+\left(1-\chi_{4}(t)\right) \dot{p}_{4 c}^{2} / 2
\end{aligned}
$$


Differentiating $f_{3}(t)$ and $f_{4}(t)$, we have

$$
\begin{aligned}
\dot{f}_{3}(t)= & k_{2} \psi_{2}\left(1-\kappa_{2} e^{-\eta_{2} t}\right)^{\psi_{2}-1} \kappa_{2} \eta_{2} e^{-\eta_{2} t} / 2 \geq 0 \\
\dot{f}_{4}(t)= & \varepsilon_{4} \psi_{1}\left(1-\kappa_{1} e^{-\eta_{1} t}\right)^{\psi_{1}-1} \kappa_{1} \eta_{1} e^{-\eta_{1} t} / 2+ \\
& \psi_{4}\left(1-\kappa_{4} e^{-\eta_{4} t}\right)^{\psi_{4}-1} \kappa_{4} \eta_{4} e^{-\eta_{4} t} / 2 \geq 0
\end{aligned}
$$

Then we have

$$
-f_{3}(t) s_{3}^{2} \leq-f_{3}(0) s_{3}^{2}=-\left(\varepsilon_{3} / 2-3 / 2-k_{2}\left(1-\left(1-\kappa_{2}\right)^{\psi_{2}}\right) / 2\right) s_{3}^{2}
$$

$-f_{4}(t) s_{4}^{2} \leq-f_{4}(0) s_{4}^{2}=-\left(\varepsilon_{4}\left(1-\kappa_{1}\right)^{\psi_{1}} / 2-3 / 2+\left(1-\kappa_{4}\right)^{\psi_{4}} / 2\right) s_{4}^{2}$

Construct Lyapunov function $V_{3}$ as

$$
V_{3}=\dot{p}_{4 c}^{2} / 2
$$

Calculating the time derivative of $V_{3}$ along the trajectories of (37) and considering the Assumption 2 is valid, yields

$$
\begin{aligned}
\dot{V}_{3} & =\dot{p}_{4 c} \ddot{p}_{4 c} \\
& =\dot{p}_{4 c} \tau_{4}^{-1}\left(\dot{p}_{4 d}-\dot{p}_{4 c}\right) \\
& \leq-\tau_{4}^{-1}\left|\dot{p}_{4 c}\right|^{2}+\left|\dot{p}_{4 c}\right| p_{f \max } \\
& =\tau_{4}^{-1}\left|\dot{p}_{4 c}\right|\left(-\left|\dot{p}_{4 c}\right|+\tau_{4} p_{f \max }\right)
\end{aligned}
$$

From (61), it is clear that $\dot{V}_{3}<0$ if $-\left|\dot{p}_{4 c}\right|+\tau_{4} p_{f \max }<0$, then we have

$$
\begin{gathered}
\left|\dot{p}_{4 c}\right| \leq \max \left\{\dot{p}_{4 c}(0), \tau_{4} p_{f \max }\right\}, \text { if } t>0 \\
\left|\dot{p}_{4 c}\right| \leq \tau_{4} p_{f \max }, \text { if } t \rightarrow \infty
\end{gathered}
$$

From Equation (34), it can be obtained that $\bar{p}_{3}, \bar{p}_{4}$ and $\bar{p}_{5}$ are bounded. And Considering the Assumption 1, Assumption 2, Equations (41) and (62) are valid, we have

$$
\begin{aligned}
& \left(\varepsilon_{3} / 2+k_{2} \chi_{2}(t) / 2\right) \bar{p}_{3}^{2}+\varepsilon_{4} \chi_{1}(t) \bar{p}_{4}^{2} / 2+ \\
& \chi_{3}(t) \bar{p}_{5}^{2} / 2+\dot{p}_{2 d}^{2} / 2+\dot{p}_{3 d}^{2} / 2+\dot{p}_{4 d}^{2} / 2+ \\
& \left(1-\chi_{3}(t)\right) d_{f}^{2} / 2+\left(1-\chi_{4}(t)\right) \dot{p}_{4 c}^{2} / 2 \leq c_{2}, \text { if } t>0
\end{aligned}
$$

where $c_{1}$ is a unknown positive constant.

Define the constants $\phi_{1}, \phi_{2}$ and $\phi_{1 \text { min }}$ as

$$
\left\{\begin{array}{l}
\phi_{1}=2 \min \left\{\varepsilon_{1}-1, \varepsilon_{2}-2, f_{3}(0), f_{4}(0)\right\} \\
\phi_{2}=2 \min \left\{\tau_{2}^{-1}-1, \tau_{3}^{-1}-1, \tau_{3}^{-1}-1\right\} \\
\phi_{1 \text { min }}=\min \left\{\phi_{1}, \phi_{2}\right\}
\end{array}\right.
$$

where $\min \{\bullet\}$ is the minimum function.

From Equations (58), (59), (64) and (65), Equation (55) can be rewritten as

$$
\begin{aligned}
\dot{V}_{2} & \leq-\phi_{1 \min }\left(\left(\frac{1}{2} \boldsymbol{s}^{T} \boldsymbol{s}\right)+\left(\frac{1}{2} \boldsymbol{e}^{T} \boldsymbol{e}\right)\right)+c_{1}+c_{2} \\
& =-\phi_{1 \min } V_{2}+c_{1}+c_{2}
\end{aligned}
$$

From Equation (62), we have

$$
V_{2} \leq V_{2}(0) e^{-\phi_{1 \min } t}+\left(c_{1}+c_{2}\right)\left(1-e^{\phi_{\min } t}\right) / \phi_{1 \min }, \text { if } t>0(67)
$$

Since $\phi_{1 \min }$ is a bounded constant, it can be known from Equation (67) that $V_{2}\left(t_{k}\right)$ is bounded for an arbitrary finite time $t=t_{k}$.

For the finite time $t=t_{k}$, the parameters are chosen as

$$
\left\{\begin{array}{l}
\varepsilon_{1}-1>\phi_{3} / 2, \varepsilon_{2}-2>\phi_{3} / 2 \\
f_{3}\left(t_{k}\right)>\phi_{3} / 2, f_{4}\left(t_{k}\right)>\phi_{3} / 2 \\
\tau_{2}^{-1}-1>\phi_{4} / 2, \tau_{3}^{-1}-1>\phi_{4} / 2, \tau_{4}^{-1}-1>\phi_{4} / 2
\end{array}\right.
$$

Where the constants $\phi_{3}>0$ and $\phi_{4}>0$. Define the constants $\phi_{2 \min }$ as

$$
\phi_{2 \min }=\min \left\{\phi_{3}, \phi_{4}\right\}>0
$$

Considering Equations (56), (57) and (68), Equation (55) can be rewritten as

$$
\begin{aligned}
\dot{V}_{2} \leq & -\phi_{2 \min }\left(\frac{1}{2} \boldsymbol{s}^{T} \boldsymbol{s}+\frac{1}{2} \boldsymbol{e}^{T} \boldsymbol{e}\right)+\left(\varepsilon_{3} / 2+k_{2} \chi_{2}(t) / 2\right) \bar{p}_{3}^{2} \\
& +\varepsilon_{4} \chi_{1}(t) \bar{p}_{4}^{2} / 2+\chi_{3}(t) \bar{p}_{5}^{2} / 2+\dot{p}_{2 d}^{2} / 2+\dot{p}_{3 d}^{2} / 2 \\
& +\dot{p}_{4 d}^{2} / 2+\left(1-\chi_{3}(t)\right) d_{f}^{2} / 2+\left(1-\chi_{4}(t)\right) \dot{p}_{4 c}^{2} / 2, \text { if } t \geq t_{k}
\end{aligned}
$$

Define the constants $\phi_{3 \max }$ as

$$
\phi_{3 \max }=\max \left\{\varepsilon_{3} / 2+k_{2} \chi_{2}(t) / 2, \varepsilon_{4} \chi_{1}(t) / 2, \chi_{3}(t) / 2\right\}
$$


Then Equation (70) can be rewritten as

$$
\begin{aligned}
\dot{V}_{2} \leq & -\phi_{2 \min }\left(\frac{1}{2} \boldsymbol{s}^{T} \boldsymbol{s}+\frac{1}{2} \boldsymbol{e}^{T} \boldsymbol{e}\right)+\phi_{3 \max }\|\overline{\boldsymbol{p}}\|+\left\|\boldsymbol{p}_{f}\right\| / 2+ \\
& \left(1-\chi_{3}(t)\right) d_{f}^{2} / 2+\left(1-\chi_{4}(t)\right) \dot{p}_{4 c}^{2} / 2, \text { if } t \geq t_{k}
\end{aligned}
$$

From Equation (31), (41), Assumption 1, Assumption 2 and Equation (72) can be rewritten as

$$
\begin{aligned}
\dot{V}_{2} \leq & -\phi_{2 \min } V_{2}+\phi_{3 \max }\|\overline{\boldsymbol{p}}\|+p_{f \max } / 2+\left(1-\chi_{3}(t)\right) d_{\max }^{2} / 2 \\
& +\left(1-\chi_{4}(t)\right)\left(\tau_{4} p_{f \max }\right)^{2} / 2, \text { if } t \geq t_{k}
\end{aligned}
$$

Then we have

$$
\dot{V}_{2} \leq-\phi_{2 \min }\left(V_{2}-\bar{V}(t)\right) \text {, if } t \geq t_{k}
$$

where

$$
\bar{V}=\left(\begin{array}{l}
\phi_{3 \max }\|\overline{\boldsymbol{p}}\|+p_{f \max } / 2+\left(1-\chi_{3}(t)\right) d_{\text {max }}^{2} / 2 \\
+\left(1-\chi_{4}(t)\right)\left(\tau_{4} p_{f \text { max }}\right)^{2} / 2,
\end{array}\right) / \phi_{2 \text { min }}
$$

Equation (74) implies that $\dot{V}_{2}<0$ if $V_{2}>\bar{V}$ and $t \geq t_{k}$. Considering $V_{2}\left(t_{k}\right)$ is bounded, it can be known that $V_{2}$ will converge to following region if $t \rightarrow \infty$ :

$$
V_{2}<\bar{V}, \text { if } t \rightarrow \infty
$$

Considering $\quad \chi_{i}(t) \rightarrow 1(i=1,2,3,4) \quad$ if $\quad t \rightarrow \infty \quad$ and Equation (31) is valid, then we have

$$
\bar{V}=\left(\phi_{3 \max } \bar{d}_{\max } /\left(c_{0}-\bar{k}\right)+p_{f \max } / 2\right) / \phi_{2 \min }, \text { if } t \rightarrow \infty
$$

Combining Equation (75) with Equation (68), then we have

$$
V_{2}<\left(\phi_{3 \max } \bar{d}_{\max } /\left(c_{0}-\bar{k}\right)+p_{f \max } / 2\right) / \phi_{2 \min }, \text { if } t \rightarrow \infty
$$

If large enough $c_{0}$ is chosen, then $d_{\max } /\left(c_{0}-\bar{k}\right)$ is small enough. If small enough $\tau_{i}(i=2,3,4)$ and large enough $\varepsilon_{i}(i=2,3,4)$ are chosen, then $\phi_{2 \min }$ is large enough (see Equation (68)). And $p_{f \max }$ is constants. Thus, it can be known from (77) that the bounded range can be made arbitrarily small through choosing large enough $c_{0}$ and small enough $\tau_{i}(i=2,3,4)$.

Remark 3.4: From the proof of Theorem 2, it is clear that the stability and control precision are not affected by the proposed SSF.

Remark 3.5: From the above research in section 3.1 and 3.2, the three purposes of this paper mentioned in section 2.2 are achieved: 1) From (38), it is clear that the proposed controller only needs the same measurement states as RRM; 2) By designing a novel SSF, the spike problem can be solve, and the control effect also will be discussed in the following simulation section; 3) Since HGO (16) estimates not only the unmeasurable state $p_{i}(i=1,2,3,4)$ but also the uncertainty $d_{f}$, the roust of controller can be guaranteed without using additional complex method.

\section{Simulation Results}

In this section, to illustrate the effectiveness of the proposed control scheme, the mathematical simulation is presented. Consider the parameters for FJMCS system (1) are selected as

$$
M g L=5, I=1, J=1, K=15
$$

The initial states are set as $\left[\begin{array}{llll}q & \dot{q} & \theta & \dot{\theta}\end{array}\right]^{T}=\left[\begin{array}{llll}0 & 0 & 0 & 0\end{array}\right]^{T}$. In addition, the maximum limit of the control input is selected as $|u| \leq 500 \mathrm{Nm}$.

Case1 (The effectiveness of SSF): This case shows that the effectiveness of the proposed SSF. The unmodeled dynamics and the disturbance from the environment are chosen as

$$
\begin{aligned}
\Delta I=0.1 I, \Delta K & =0.1 K, \Delta M g L=0.1 M g L, \\
d_{1}=d_{2}= & 0.1 \sin (t / 3)
\end{aligned}
$$

The parameters of controller (38) are chosen as

$$
\varepsilon_{1}=2, \varepsilon_{2}=2, \varepsilon_{3}=7, \varepsilon_{4}=12, \tau_{2}=\tau_{3}=\tau_{4}=0.01
$$

The desired rotation angle of link is chosen as

$$
q_{d}=1 \mathrm{rad}
$$

To ensure the estimation error is small enough, a high observer gain of HGO is selected as

$$
\omega_{0}=100
$$

The initial value of HGO is set as

$$
\left[\begin{array}{lllll}
\hat{p}_{1} & \hat{p}_{2} & \hat{p}_{3} & \hat{p}_{4} & \hat{p}_{5}
\end{array}\right]^{T}=\left[\begin{array}{lllll}
0 & 0 & 0 & 0 & 0
\end{array}\right]^{T}
$$


It is clear that $\hat{p}_{1}(0) \neq p_{1}(0)$. Consider the following two control schemes in Case 1:

(1) controller (38) without SSF: $\quad \chi_{i}(t)=0, i=1,2,3,4$. (2) controller (38) with SSF : $\chi_{i}(t)=\left(1-0.97 e^{-4.5}\right)^{5}, i=1,2,3,4$.

Figures 2 9 show the simulation results for case1. From Figures 2, for the controller without SSF, it is clear that the tracking error converges slowly and an undesirable large overshoot phenomenon can be observed. As mentioned before, the reason is that the spike problem of HGO can be caused by choosing a large gain if $p_{1}(0) \neq \hat{p}_{1}(0)$. And the spike values from $\mathrm{HGO}$ can be taken into the control input, directly or indirectly. The spike values from HGO can be observed from Figures 4 8. And Figures 3 shows that the control input of the controller without SSF is saturated under the spike value. From Figures $2 \sim 3$, it is also clear that the spike values can be suppressed effectively by the proposed controller with SSF. An excellent control performance still can be guaranteed by the proposed controller with SSF. From Figure 9, it can be seen that the SSF $\chi_{i}(t)$ is small enough in the initial time, thus the spike values can be suppressed. And $\chi_{i}(t) \rightarrow 1$ if the time is large enough, then control precision will not be affected by the SSF.

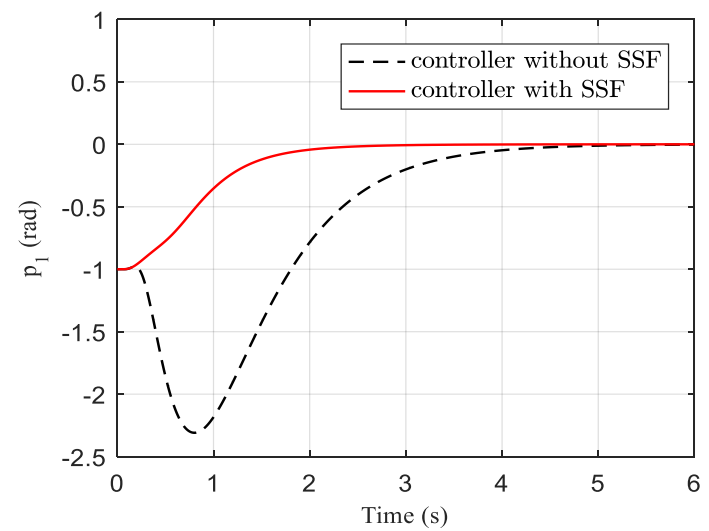

Figure 2 Tracking error $p_{1}$ (Case 1)

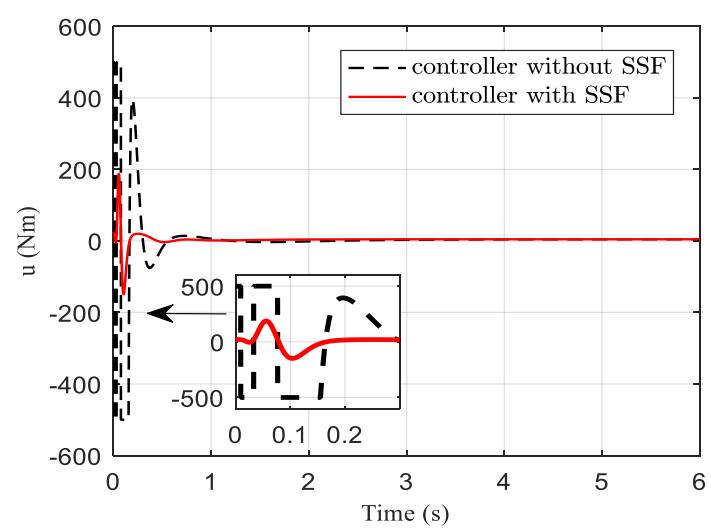

Figure 3 Control input $u$ (Case 1)

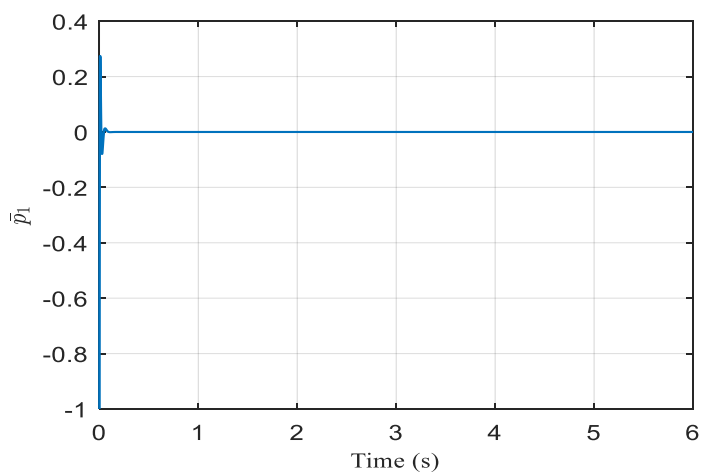

Figure 4 Estimate error $\bar{p}_{1}$ (Case 1)

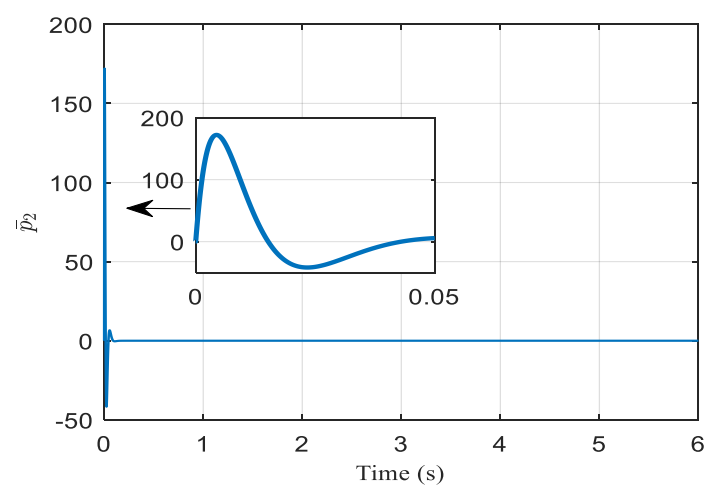

Figure 5 Estimate error $\bar{p}_{2}$ (Case 1)

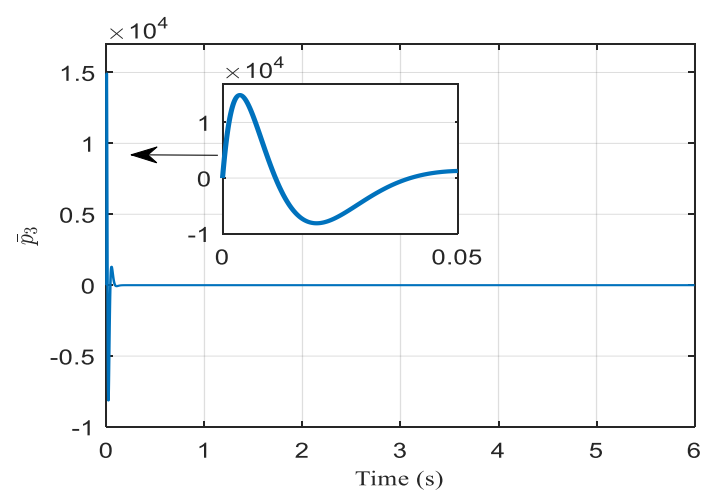

Figure 6 Estimate error $\bar{p}_{3}$ (Case 1)

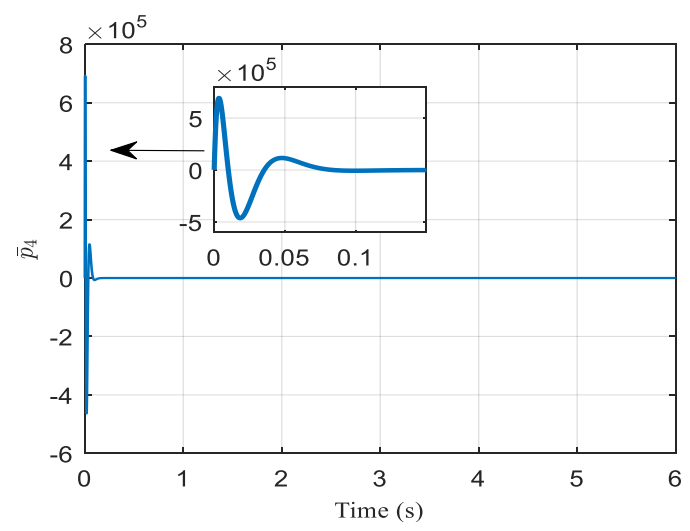

Figure 7 Estimate error $\bar{p}_{4}$ (Case 1) 


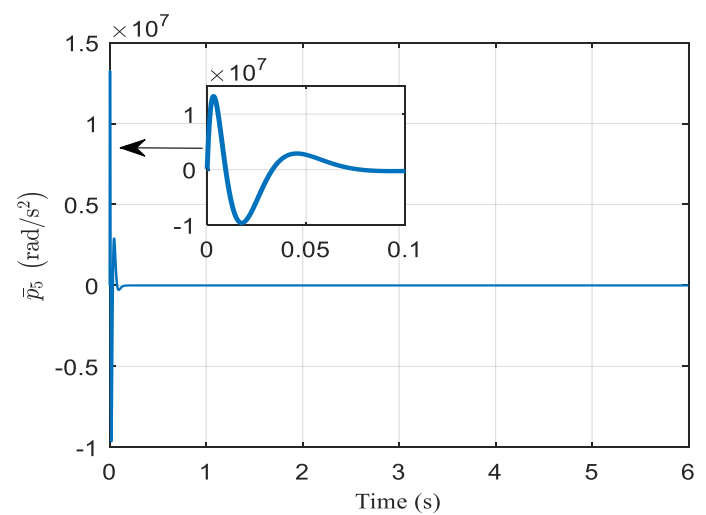

Figure 8 Estimate error $\bar{p}_{5}$ (Case 1)

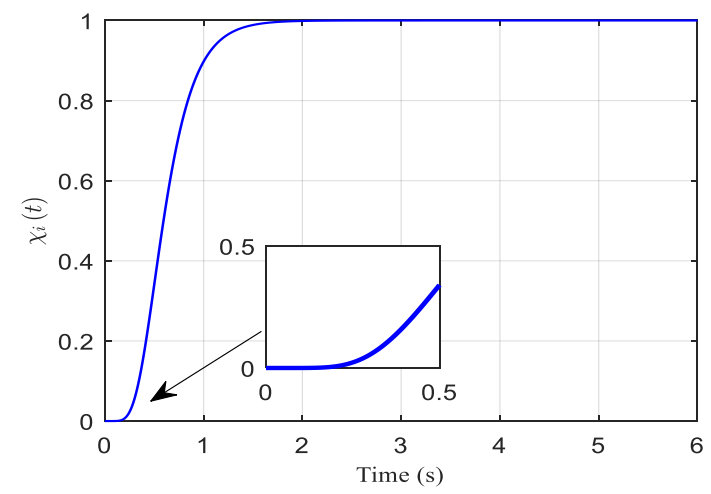

Figure $9 \operatorname{SSF} \chi_{i}(t)$

Case2 (The control performance of partial states feedback): To shows the performance of the proposed partial states feedback scheme, the following two controllers are compared in this case:

(1) Proposed partial state feedback controller (PSFC): Like case 1, the expression of controller is given in (38). The parameters of PSFC and observer are given in (80) and (82). And the SSF also is chosen as $\chi_{i}(t)=\left(1-0.97 e^{-4.5}\right)^{5}, i=1,2,3,4$.

(2) Full state feedback controller (FSFC): It is assumed that all states are measurable, which means that it is unnecessary to design the observer to estimate the unmeasurable states. Then, only based on the conventional DSM [22-24], the FSFC for FJMCS (4) can be designed as

$$
\left\{\begin{array}{l}
u=b^{-1}\left(-\varepsilon_{x 4}\left(x_{4}-x_{4 c}\right)-k_{1}\left(x_{1}+q_{d}\right)-k_{2} x_{3}-\right. \\
\left.\quad \sigma_{2} \operatorname{sigmf}\left(x_{4}-x_{4 c}\right)+\dot{x}_{4 c}\right) \\
x_{2 d}=-\varepsilon_{x 1}\left(x_{1}-q_{d}\right) \\
\tau_{x 2} \dot{x}_{2 c}+x_{2 c}=x_{2 d} \\
x_{3 d}=-\varepsilon_{x 2}\left(x_{2}-x_{2 c}\right)-f_{1}\left(x_{1}\right)+\dot{x}_{2 c}-\sigma_{1} \operatorname{sigmf}\left(x_{2}-x_{2 c}\right) \\
\tau_{x 3} \dot{x}_{3 c}+x_{3 c}=x_{3 d} \\
x_{4 d}=-\varepsilon_{x 3}\left(x_{3}-x_{3 c}\right)+\dot{x}_{3 c} \\
\tau_{x 4} \dot{x}_{4 c}+x_{4 c}=x_{4 d} \\
\operatorname{sigmf}(x)=2\left(\frac{1}{1+e^{-20 x}}-\frac{1}{2}\right)
\end{array}\right.
$$

Where the parameters are chosen as

$$
\begin{aligned}
& \varepsilon_{x 1}=2, \varepsilon_{x 2}=2, \varepsilon_{x 3}=7, \varepsilon_{x 4}=12, \tau_{x 2}=0.1, \\
& \tau_{x 3}=0.05, \tau_{x 4}=0.03, \sigma_{1}=1, \sigma_{2}=400
\end{aligned}
$$

And $\operatorname{sigmf}(\bullet)$ is used to avoid the chattering problem and suppress the uncertainties. The explanation of $\operatorname{sigmf}(\bullet)$ can be found in previous work ${ }^{35}$. The unmodeled dynamics and the disturbance from the environment are chosen as in (79).

The above PSFC and FSFC are used for tracking two kinds of desired angles $q_{d}=1 \mathrm{rad}$ and $q_{d}=\cos (t) \operatorname{rad}$, respectively. The simulation results for case 2 are shown in the Figures 10 13. From the simulation results, it is clear that both the conventional FSFC and the proposed PSFC can guarantee the fast and accurate convergence of tracking error for the two kinds of desired angles. Thus, compared with the conventional full states feedback scheme, the proposed method of this paper can achieve a similar excellent control performance even with partial states feedback.

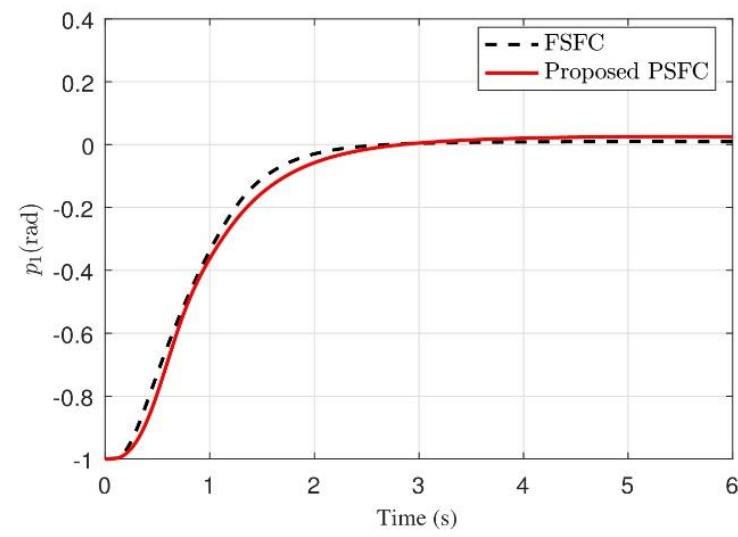

Figure 10 Tracking error $p_{1}\left(\right.$ case 2: $\left.q_{d}=1 \mathrm{rad}\right)$

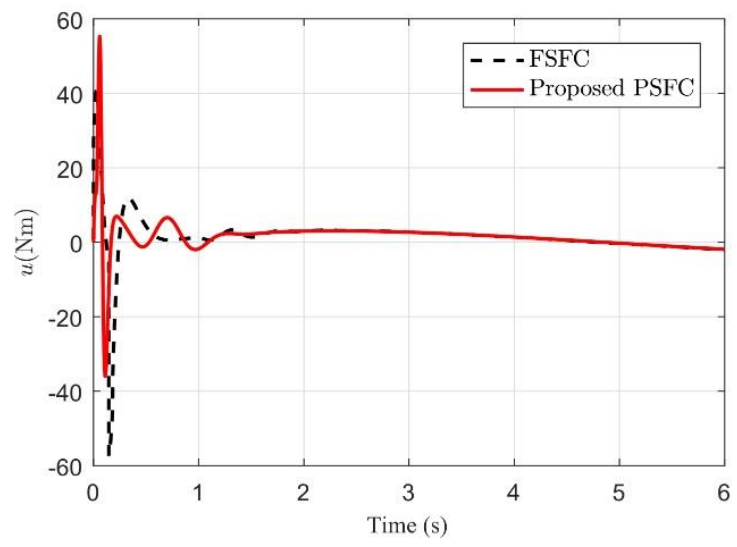

Figure 11 Control input $u$ (case 2: $\left.q_{d}=1 \mathrm{rad}\right)$ 


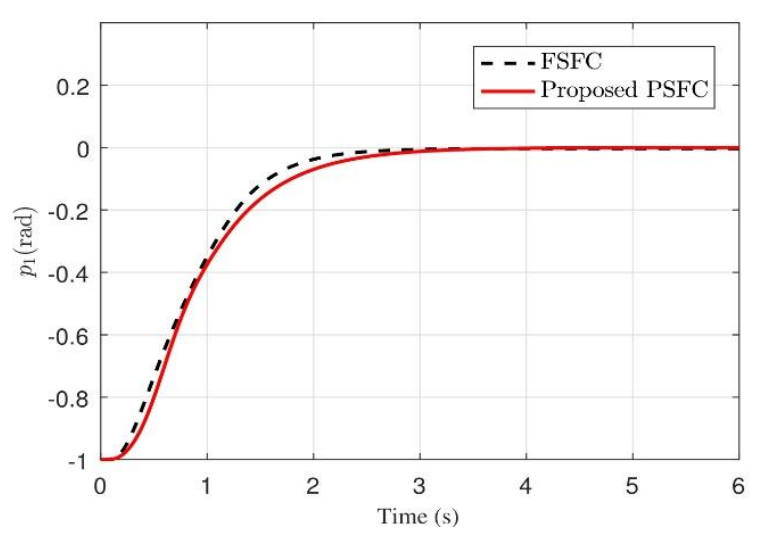

Figure 12 Tracking error $p_{1}\left(\right.$ case 2: $\left.q_{d}=\cos (t) \operatorname{rad}\right)$

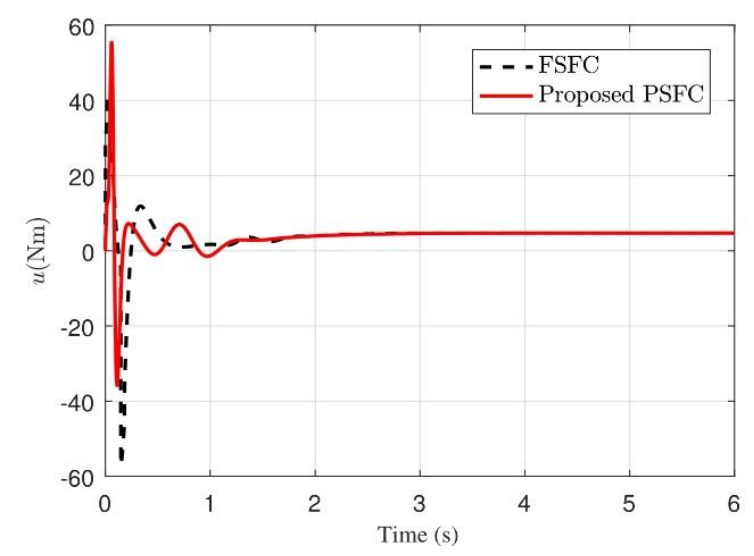

Figure 13 Control output $u$ (case 2: $\left.q_{d}=\cos (t) \mathrm{rad}\right)$

Case3 (The anti-disturbance performance of the proposed partial states feedback scheme): To show the anti-disturbance performance of the propose scheme, the following three kinds of unmodeled dynamics and disturbances from the environment are chosen as:

(1) Small amplitude and low frequency

$$
\begin{gathered}
\Delta I=0.05 I, \Delta K=0.05 K, \Delta M g L=0.05 M g L, \\
d_{1}=d_{2}=0.1 \sin (t / 6)
\end{gathered}
$$

(2) Large amplitude and low frequency

$$
\begin{gathered}
\Delta I=0.3 I, \Delta K=0.3 K, \Delta M g L=0.3 M g L, \\
d_{1}=d_{2}=\sin (t / 6)
\end{gathered}
$$

(3) Large amplitude and high frequency

$$
\begin{aligned}
& \Delta I=0.3 \sin (t) I, \Delta K=0.3 \sin (t) K, \\
& \Delta M g L=0.3 \sin (t) M g L, d_{1}=d_{2}=\sin (t)
\end{aligned}
$$

The parameters of proposed PSFC are given in Equations (84) and (86). And the SSF also is chosen as $\chi_{i}(t)=\left(1-0.97 e^{-4.5}\right)^{5}, i=1,2,3,4$.

Figures 14 15 show the simulation results for case3. From Figures 14 and 15, lt is clear that the proposed PSFC all can achieve a good anti-disturbance performance for the three kinds of unmodeled dynamics and disturbances.

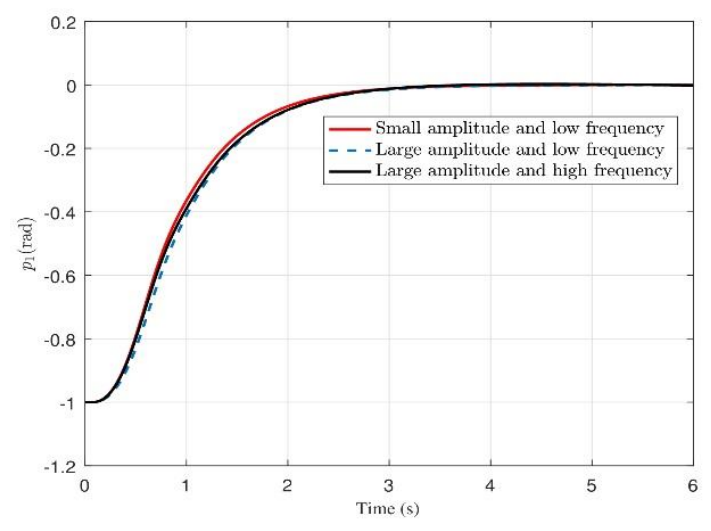

Figure 14 Tracking error $p_{1}$

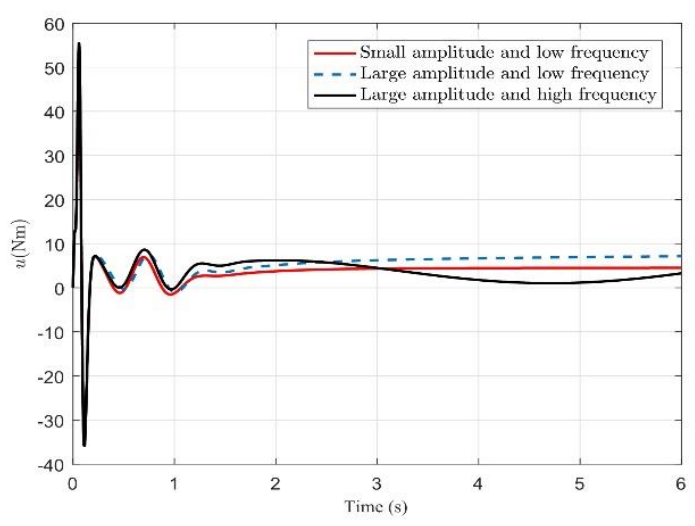

Figure 15 Control input $u$

\section{Conclusions}

In this paper, a novel PSFC is proposed for the FJM with unmeasurable system states based on DSM and HGO. The main contributions here are that:

1) The proposed method only needs the same measurement states as conventional RRM;

2) The estimation spike problem in the existing HGObased control is solved by developing a novel SSF;

3) Without using additional complex robust method which is wildly used in the conventional PSFC schemes, the proposed control scheme still can suppress the uncertainties by using HGO to estimate the unmeasurable states and uncertainties simultaneously. Finally, mathematical simulation result demonstrated that the performances of the proposed controller is excellent.

\section{Declaration}

\section{Funding}

This work is supported by Natural Science Foundation of Shaanxi Province (Grant No. 2020JM-131 and 2020KW058). This work is supported by the Key Research and 
Development Program of Shaanxi (No. 2019GY-025, 2018GY-091) and Xi'an Science and Technology Plan Project (2020KJRC0134).

\section{Availability of data and materials}

The datasets supporting the conclusions of this article are included within the article.

\section{Competing interests}

The authors declare no competing financial interests.

\section{Consent for publication}

Not applicable

\section{Ethics approval and consent to participate}

Not applicable

\section{References}

[1] Albu-Schäffer. "The DLR lightweight robot: Design and control concepts for robots in human environments." Industrial Robot-The International Journal of Robotics Research and Application. vol.34, pp.376--385, 2008.

[2] Becerra V M, Cage C N J, "Harwin W S. Hardware retrofit and computed torque control of a Puma 560 Robot updating an industrial manipulator." IEEE Control Systems Magazine. vol 24, pp.78--82, 2004.

[3] Kiang C T, Spowage A, Yoong C K. "Review of control and sensor system of flexible manipulator." Journal of Intelligent \& Robotic Systems. vol 77, pp.187--213, 2015.

[4] Chen Y, Yu R, Zhang Y, et al. "Circular formation flight control for unmanned aerial vehicles with directed network and external disturbance." IEEE/CAA Journal of Automatica Sinica. vol 7, pp.505$-516,2019$.

[5] Chang Y C, Yen H M. "Design of a robust position feedback tracking controller for flexible-joint robots." IET Control Theory \& Applications. vol 5, pp.351--363, 2011.

[6] Zhang L, Li Z, Yang C. "Adaptive neural network based variable stiffness control of uncertain robotic systems using disturbance observer." IEEE Transactions on industrial electronics. vol 64, pp.2236--2245, 2016.

[7] Amer AF, Sallam EA, and Elawady WM. "Adaptive fuzzy sliding mode control using supervisory fuzzy control for 3 DOF planar robot manipulators." Applied Soft Computing. vol 11, pp.4943--4953, 2001.

[8] Yin X, Li P. "Enhancing trajectory tracking accuracy for industrial robot with robust adaptive control." Robotics and ComputerIntegrated Manufacturing. vol 51, pp.97--102, 2018.

[9] Fei Y, Wu Q. "Tracking control of robot manipulators via output feedback linearization." Frontiers of Mechanical Engineering in China. vol 1, pp.329--335, 2006.

[10] Wang H, Pan Y, Li S, et al. "Robust sliding mode control for robots driven by compliant actuators." IEEE Transactions on Control Systems Technology. vol 27, pp.1259--1266, 2018.

[11] Kiang C T, Spowage A, Yoong C K. "Review of control and sensor system of flexible manipulator." Journal of Intelligent \& Robotic Systems. vol 77, pp.187--213, 2015.

[12] Izadbakhsh. A. "Robust control design for rigid-link flexible-joint electrically driven robot subjected to constraint:Theory and experimental verification." Nonlinear Dynamics. vol 85, pp.1--15, 2016.

[13] J. Lessard, P. Bigras, Z. Liu. "Characterization, Modeling and vibration control of a flexible joint for a robotic system." Journal of Vibration and Control. vol 20, pp.943--960, 2012.

[14] Nanos K, Papadopoulos E G. "Characterization, On the dynamics and control of flexible joint space manipulators." Control Engineering Practice. vol 45, pp.230--243, 2015.

[15] Koshkouei A J, Zinober A S I, "Burnham K J. Adaptive sliding mode backstepping control of nonlinear systems with unmatched uncertainty." Asian Journal of control. vol 6, pp.447--453, 2004.

[16] Cong B, Liu X, Chen Z. "Backstepping based adaptive sliding mode control for spacecraft attitude maneuvers." Aerospace Science and Technology. vol 30, pp.1--7, 2013.

[17] Tran D T, Ba D X, Ahn K K. "Adaptive backstepping sliding mode control for equilibrium position tracking of an electrohydraulic elastic manipulator." IEEE Transactions on Industrial Electronics. vol 67, pp.3860--3869; 2019.

[18] Wang L, Zhang D, Liu J, et al. "Adaptive fault-tolerant control for a joint flexible manipulator based on dynamic surface." Transactions of the Institute of Measurement and Control. vol 41, pp.4240--4253, 2019.

[19] Dian S, Hu Y, Zhao T, et al. "Adaptive backstepping control for flexible-joint manipulator using interval type-2 fuzzy neural network approximator." Nonlinear Dynamics. vol 97, pp.1567--1580, 2019.

[20] Sahab A R, Modabbernia M R. "Backstepping method for a singlelink flexible-joint manipulator using genetic algorithm." Int. J. Innov. Comput. Inf. Control. vol 7, pp.4161--4170,2011.

[21] Chu M, Jia Q, Sun H. "Backstepping control for flexible joint with friction using wavelet neural networks and L2-gain approach." Asian Journal of Control. vol 20, pp.856--866, 2018.

[22] Xin L P, Yu B, Zhao L, et al. "Adaptive fuzzy backstepping control for a two continuous stirred tank reactors process based on dynamic surface control approach." Applied Mathematics and Computation. vol 377, pp.125--138, 2020.

[23] Sun G, Wang D, Li X, et al. "A DSC approach to adaptive neural network tracking control for pure-feedback nonlinear systems." Applied Mathematics and Computation. vol 219, pp.6224--6235, 2013.

[24] Wang L, Zhang D, Liu J, et al. "Adaptive fault-tolerant control for a joint flexible manipulator based on dynamic surface." Transactions of the Institute of Measurement and Control. vol 41, pp.4240--4253, 2019.

[25] Chen M, Ge SS. "Adaptive neural output feedback control of uncertain nonlinear systems with unknown hysteresis using disturbance observer." IEEE Transactions on Industrial Electronics. vol 62, pp.7706--7716, 2015 .

[26] Tong S, Sui S, Li Y. "Fuzzy adaptive output feedback control of MIMO nonlinear systems with partial tracking errors constrained." IEEE Transactions on Fuzzy Systems. vol 23, pp.729--742, 2015.

[27] Hu J, Sun X, He L. "Adaptive output feedback formation tracking for a class of multiagent systems with quantized input signals." Frontiers of Information Technology \& Electronic Engineering. vol 19, pp.1086--1097, 2018.

[28] Wang H, Zhang Q, Xian J, et al. "Robust Finite-Time Output Feedback Control for Systems with Unpredictable Time-Varying Disturbances." IEEE Access. vol 8, pp.52268--52277, 2020. 
[29] Yoo SJ, Park JB, Choi YH. "Output feedback dynamic surface control of flexible-joint robots." International Journal of Control Automation and Systems. vol 6, pp.223--233, 2008.

[30] Liu H, Huang Y. "Robust adaptive output feedback tracking control for flexible-joint robot manipulators based on singularly perturbed decoupling." Robotica. vol 36, pp.822, 2018.

[31] Liu H S, Huang Y. "Bounded adaptive output feedback tracking control for flexible-joint robot manipulators." Journal of Zhejiang University-SCIENCE A. vol 19, pp.557--578, 2018.

[32] Zhao Z L, Guo B Z. "On active disturbance rejection control for nonlinear systems using time-varying gain." European Journal of Control. vol 23, pp.62--70, 2015.

[33] Pu Z, Yuan R, Yi J, et al. "A class of adaptive extended state observers for nonlinear disturbed systems." IEEE Transactions on Industrial Electronics. vol 62, pp.5858--5869, 2015.

[34] Liu X, Yang C, Chen Z, et al. "Neuro-adaptive observer-based control of flexible joint robot.” Neurocomputing. vol 275, pp.73--82, 2018.

[35] Cho D, Kim H J, Tahk M J. "Nonsingular sliding mode guidance for impact time control." Journal of Guidance, Control, and Dynamics. vol 39, pp.61--68, 2016.

\section{Biographical notes}

De-Zhi Kong, received the B.S. degree in 2014 from Northeastern University, China. He received M.S. degree in 2017 from University of Sydney, Australia. He is currently a doctoral candidate of Northwestern Polytechnical University. His main research pursuit is robot control, nonlinear control theory .et.

Tel: +86-18629258478; E-mail: dzkong@mail.nwpu.edu.cn

Wen-Dong Wang, received the B.S degree in Mechanical Engineering and Automation from Shandong Jianzhu University, Jinan, in 2008 and the M.S and Ph.D. degree in Mechatronics from Northwestern Polytechnical University, Xi'an, China, in 2010 and 2014, where he has been an associate Professor with the School of Mechanical Engineering since 2018. From 2011 to 2013, he was a research assistant in Robot and Automation Laboratory, University of Toronto, Canada. He has authored or co-authored more than 20 technical papers in international journals and conferences. His research interests include the design and control algorithm of rehabilitation and massage robot, human-robot interaction and application of artificial intelligence for healthcare robot.et.

E-mail: wdwang@nwpu.edu.cn

Yi-Kai Shi, is professor and doctoral supervisor of the School of Mechanical and Electrical Engineering of Northwestern Polytechnical University. Mainly engaged in the research of electronic mechanical engineering and electrical engineering .et.

E-mail: ykshi@nwpu.edu.cn 
Figures

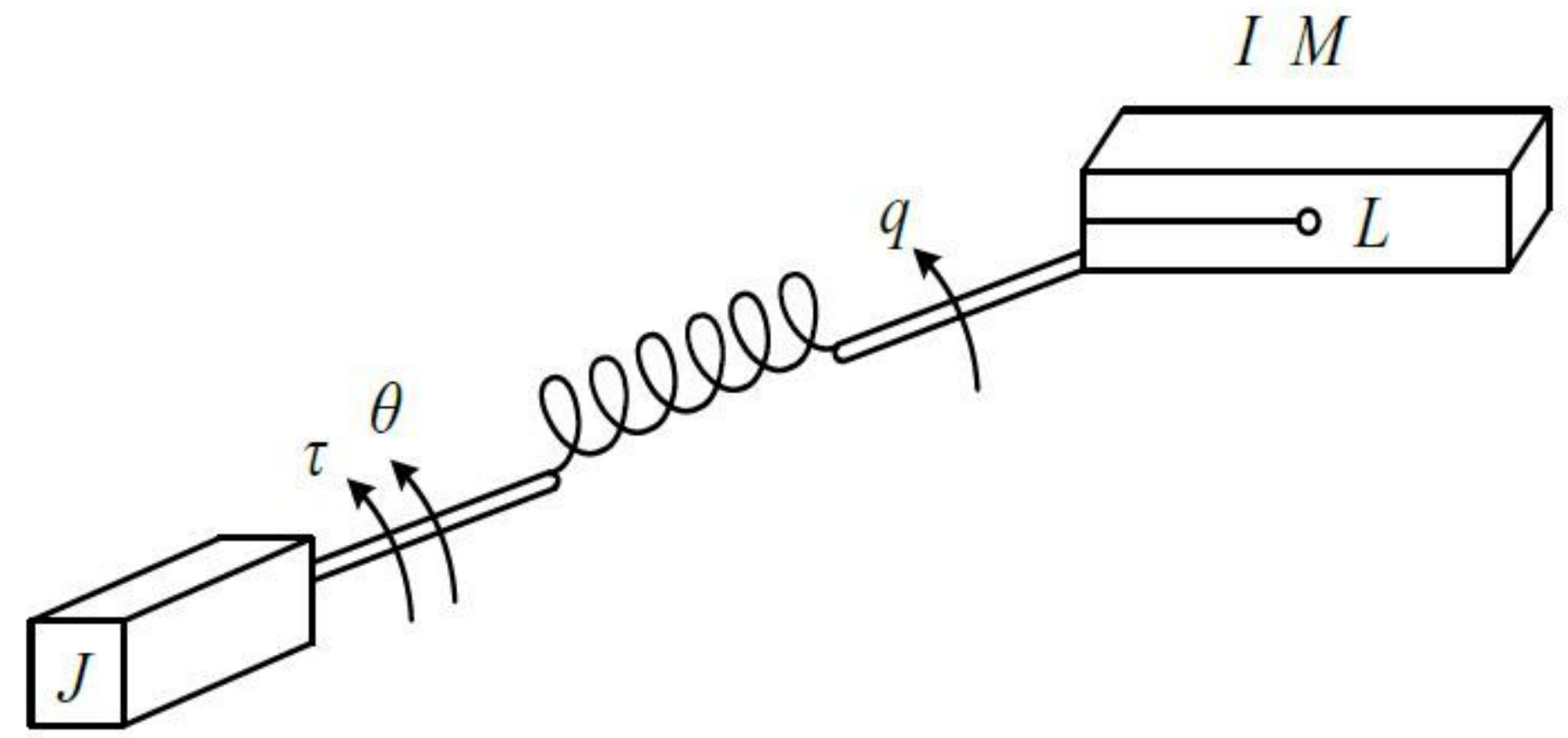

Figure 1

Structure of flexible manipulator

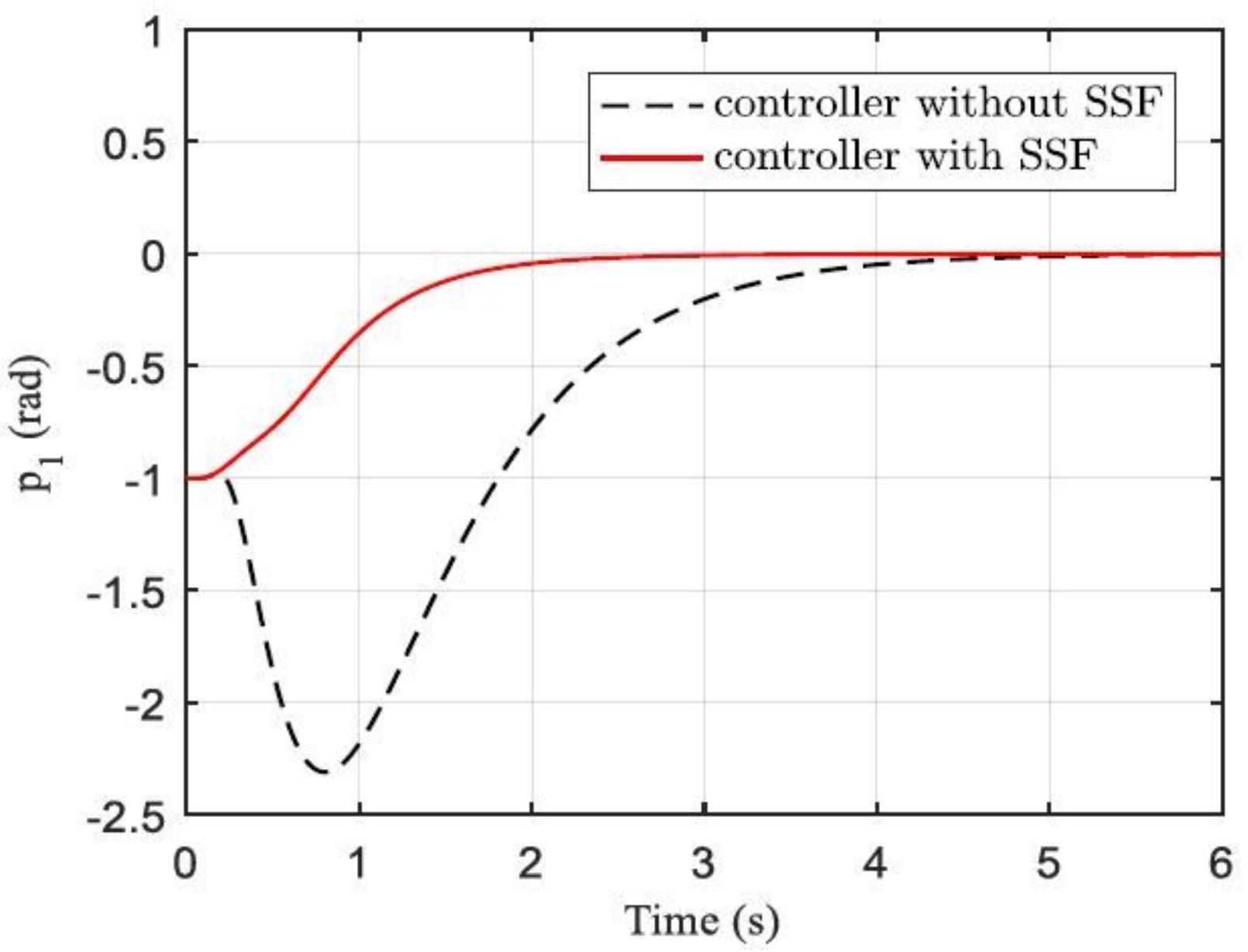


Figure 2

Tracking error P1 (Case 1)

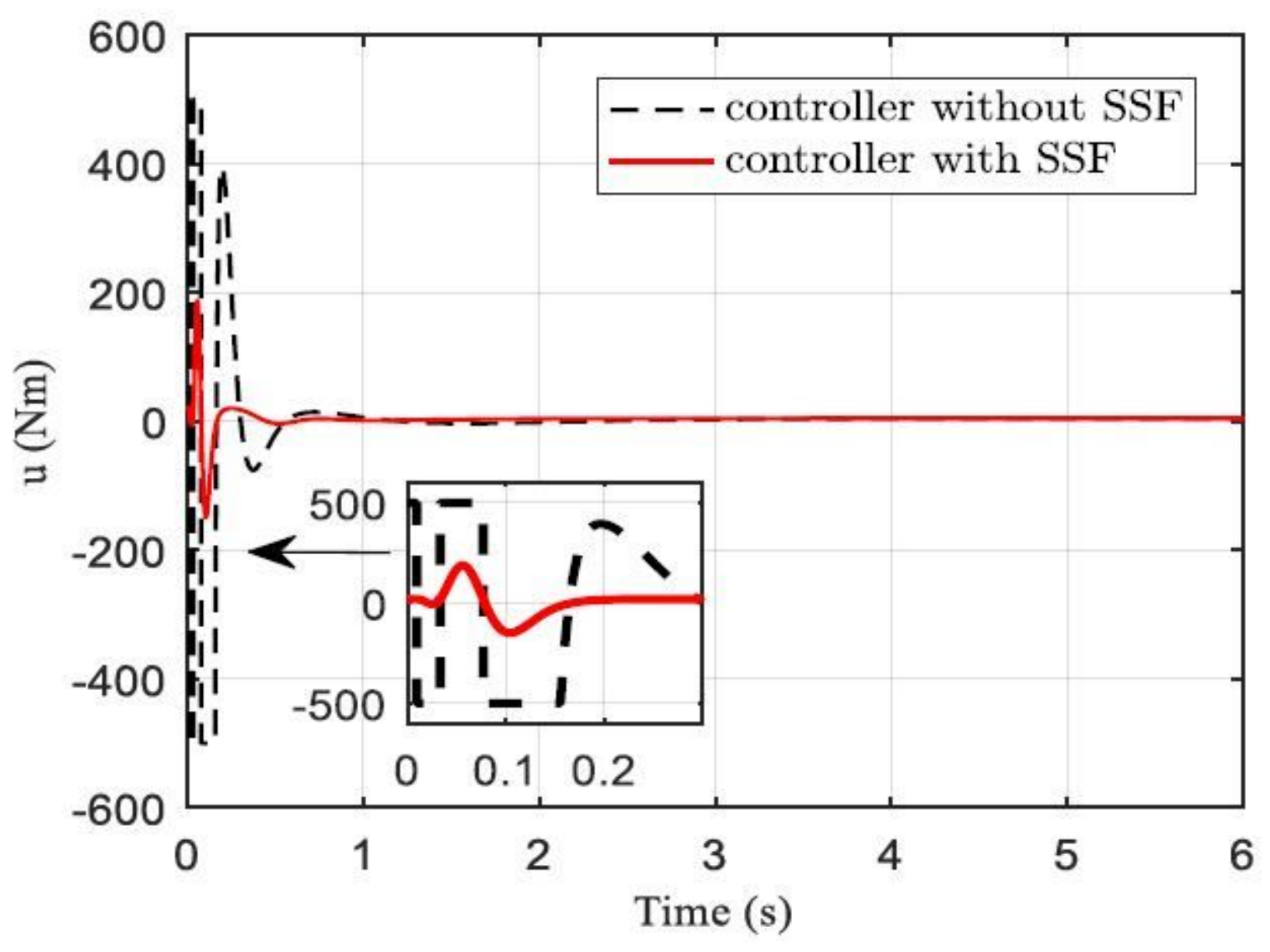

Figure 3

Control input u (Case 1) 


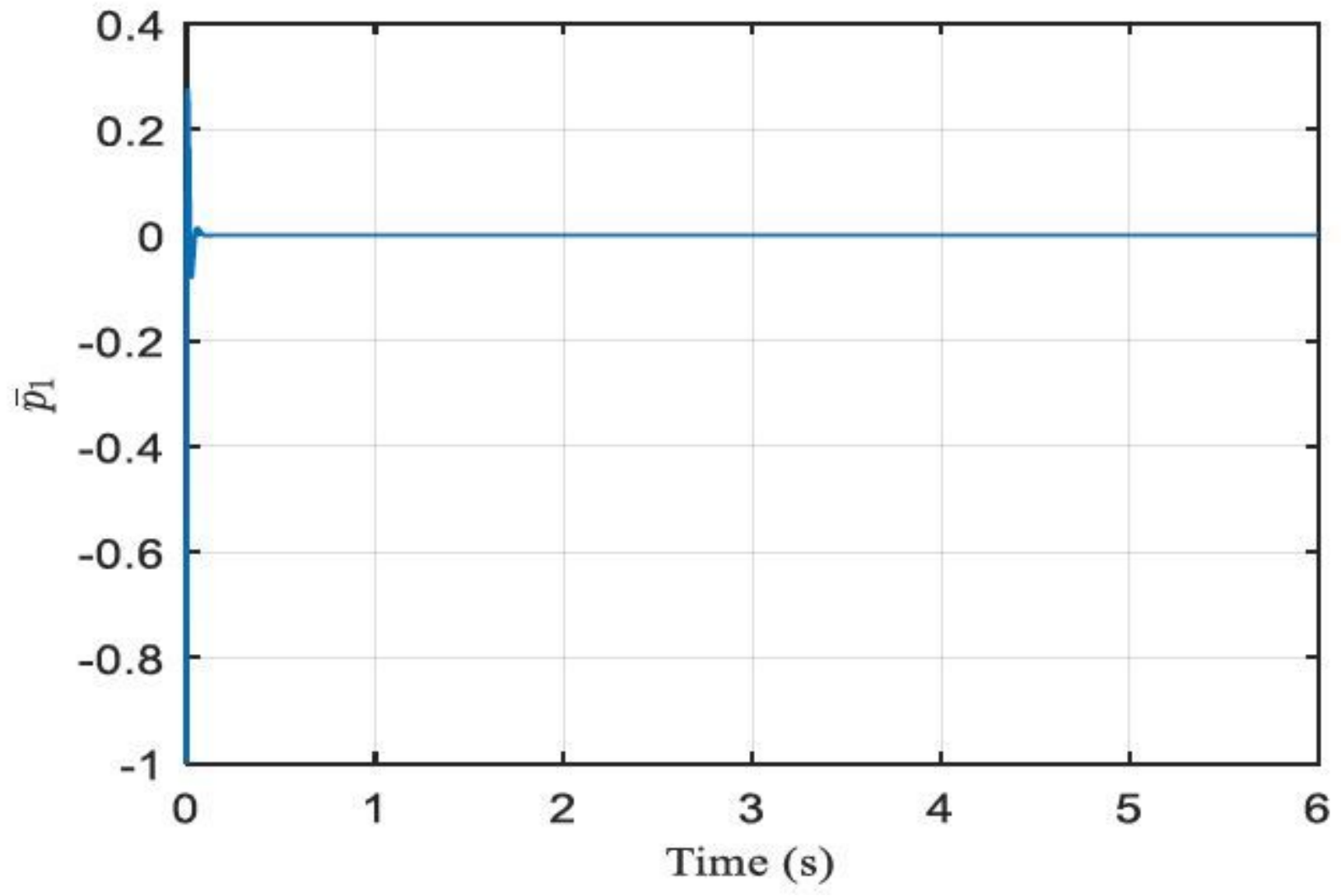

Figure 4

Estimate error P1 (Case 1) 


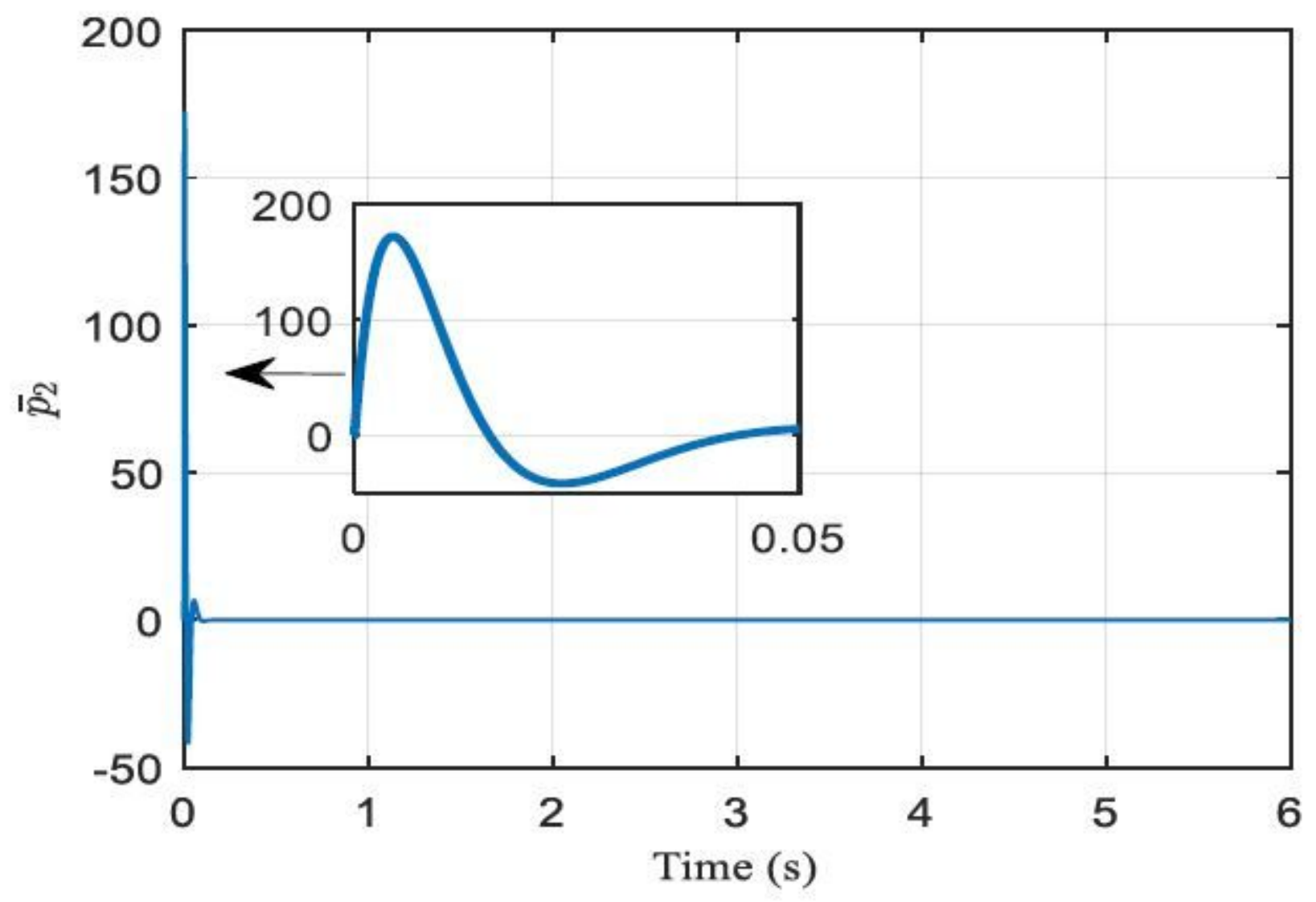

Figure 5

Estimate error P2 (Case 1) 


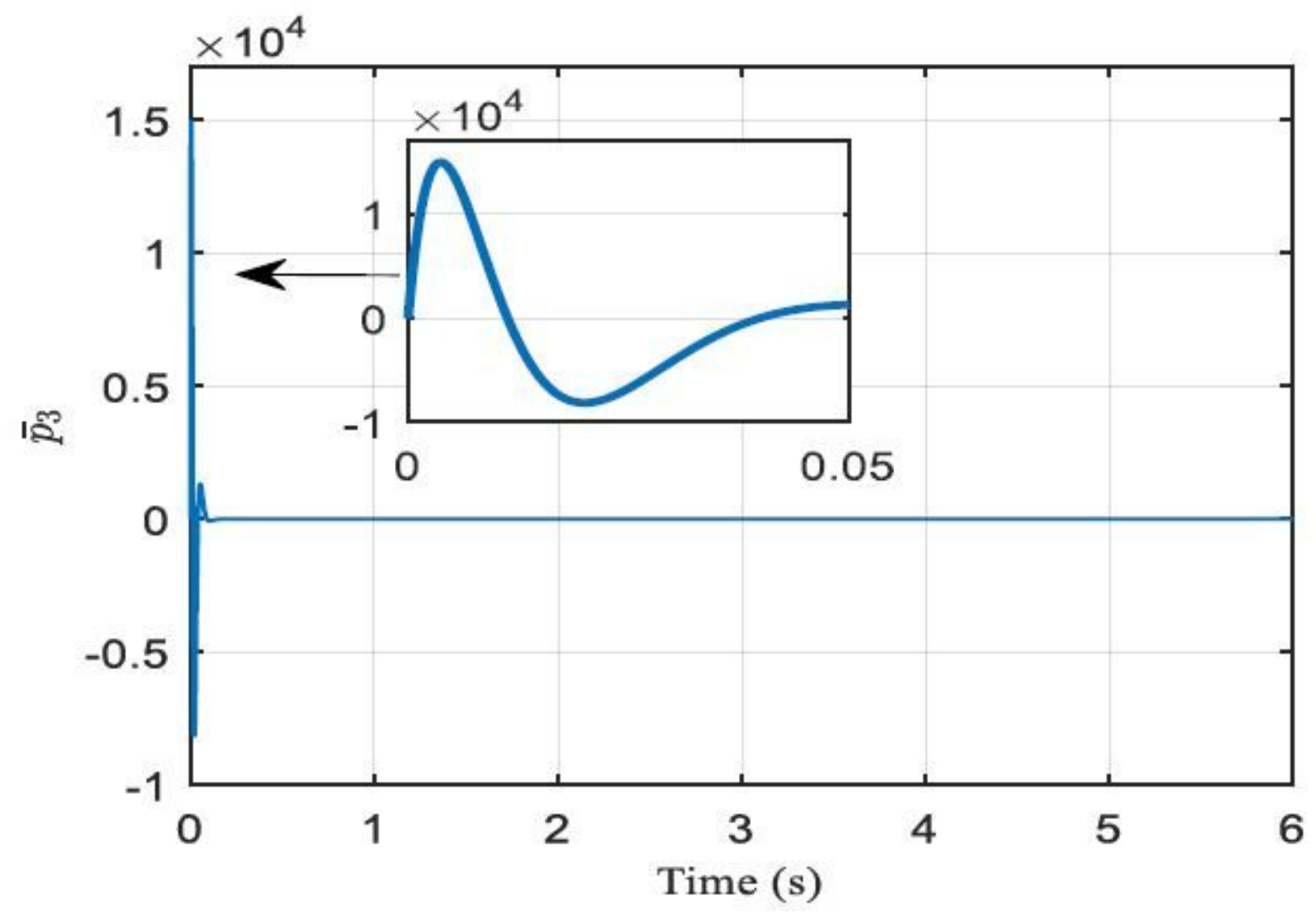

Figure 6

Estimate error P3 (Case 1) 


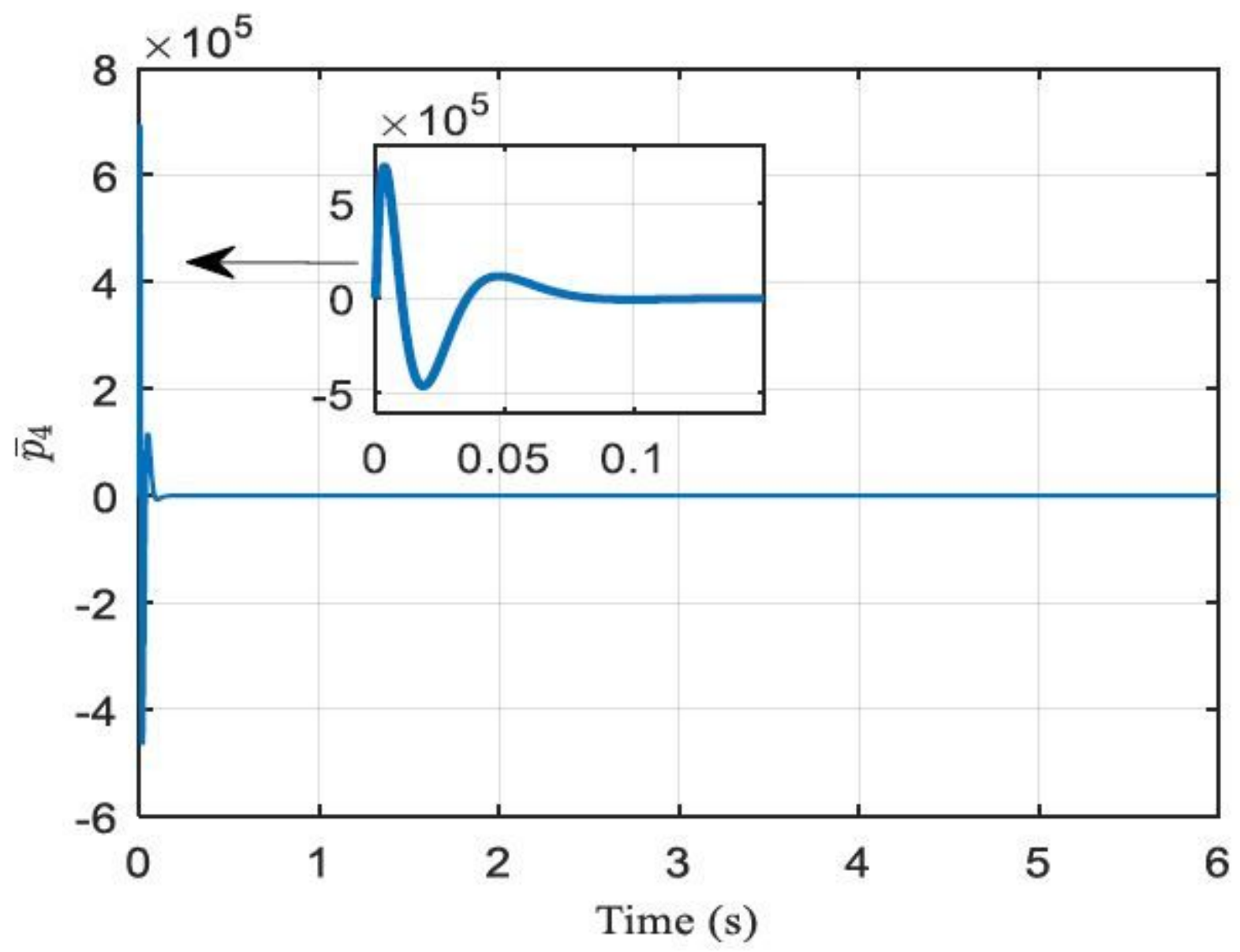

Figure 7

Estimate error P4 (Case 1) 


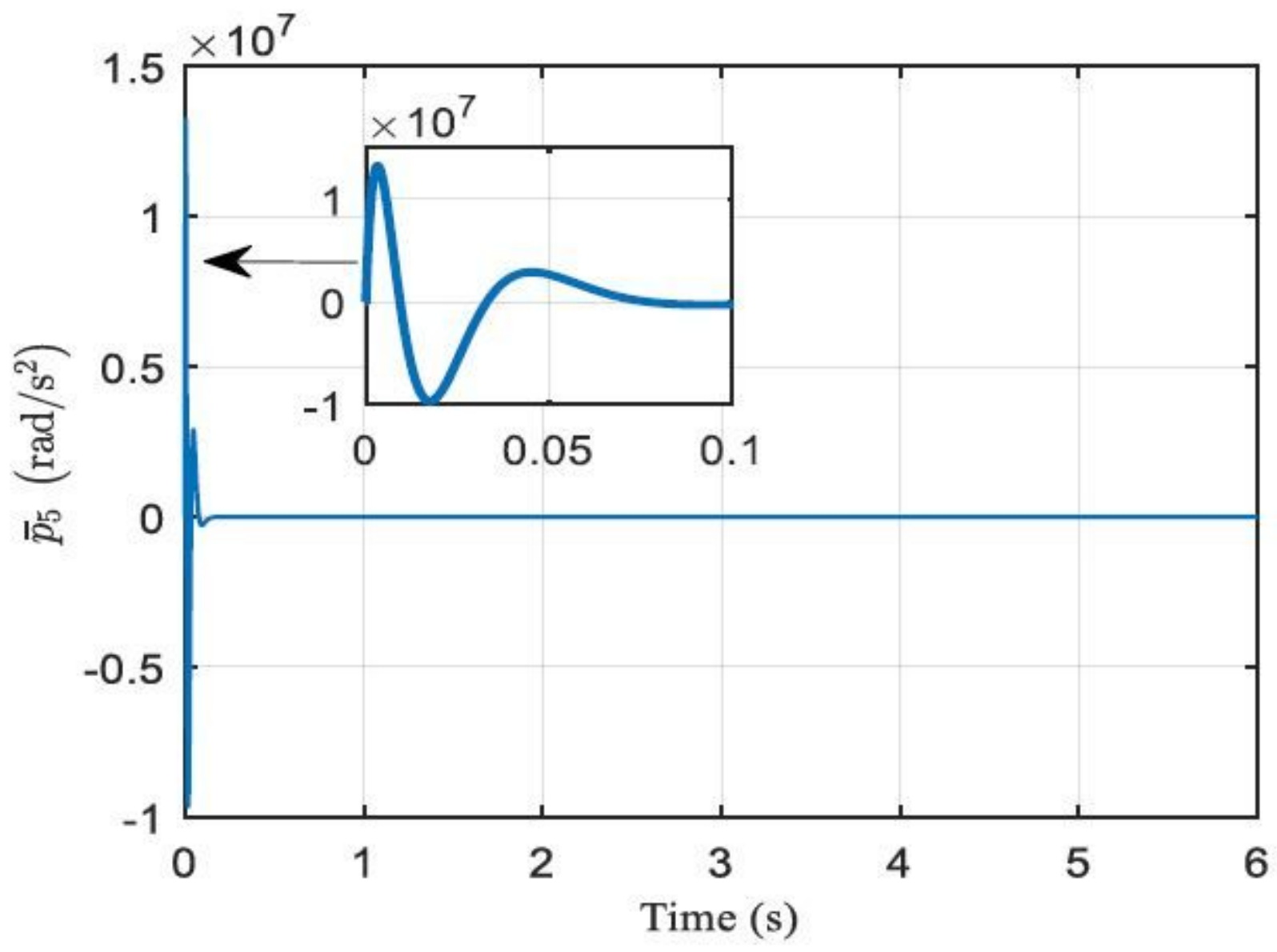

Figure 8

Estimate error P5 (Case 1) 


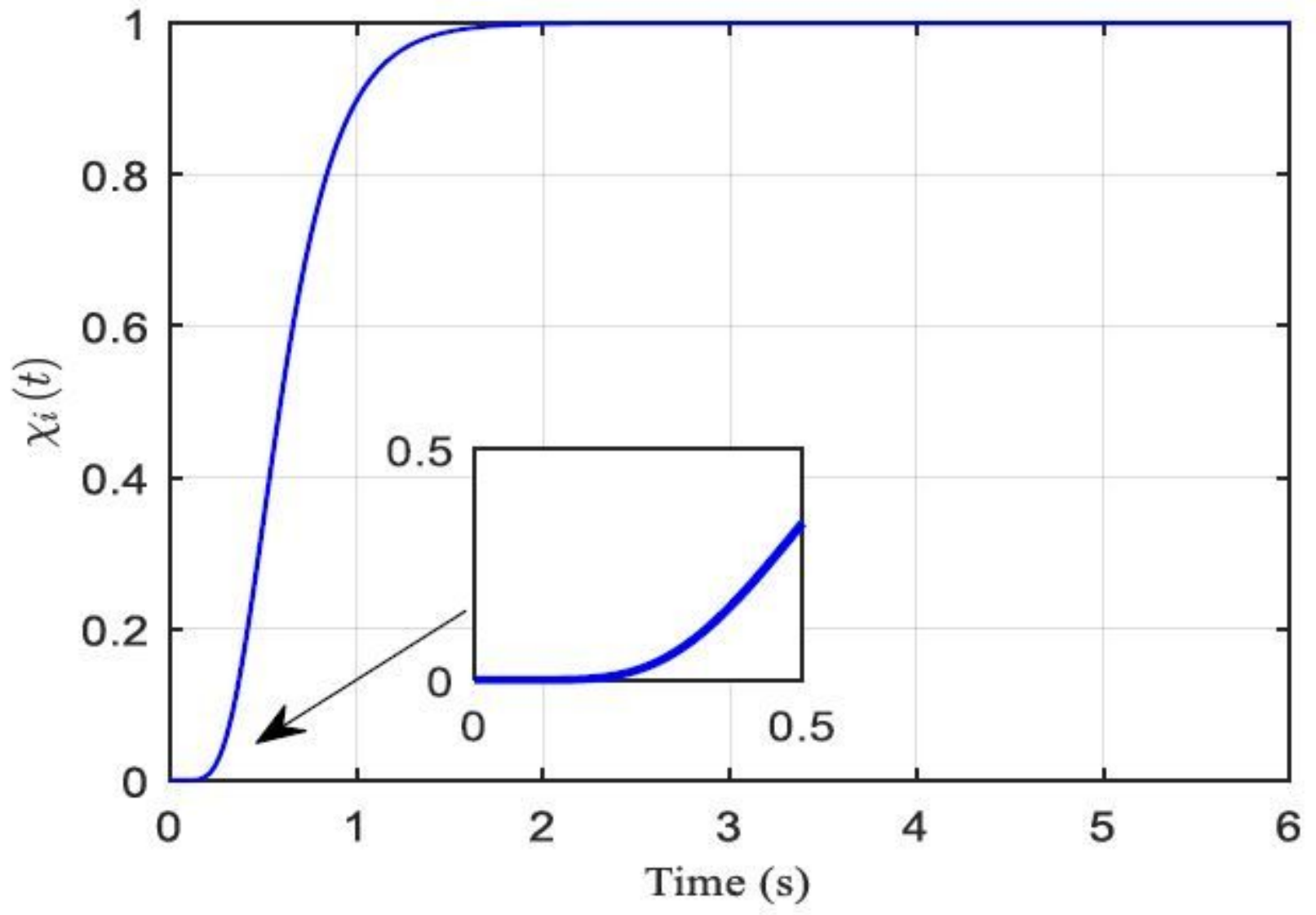

Figure 9

SSF Xi (t) 


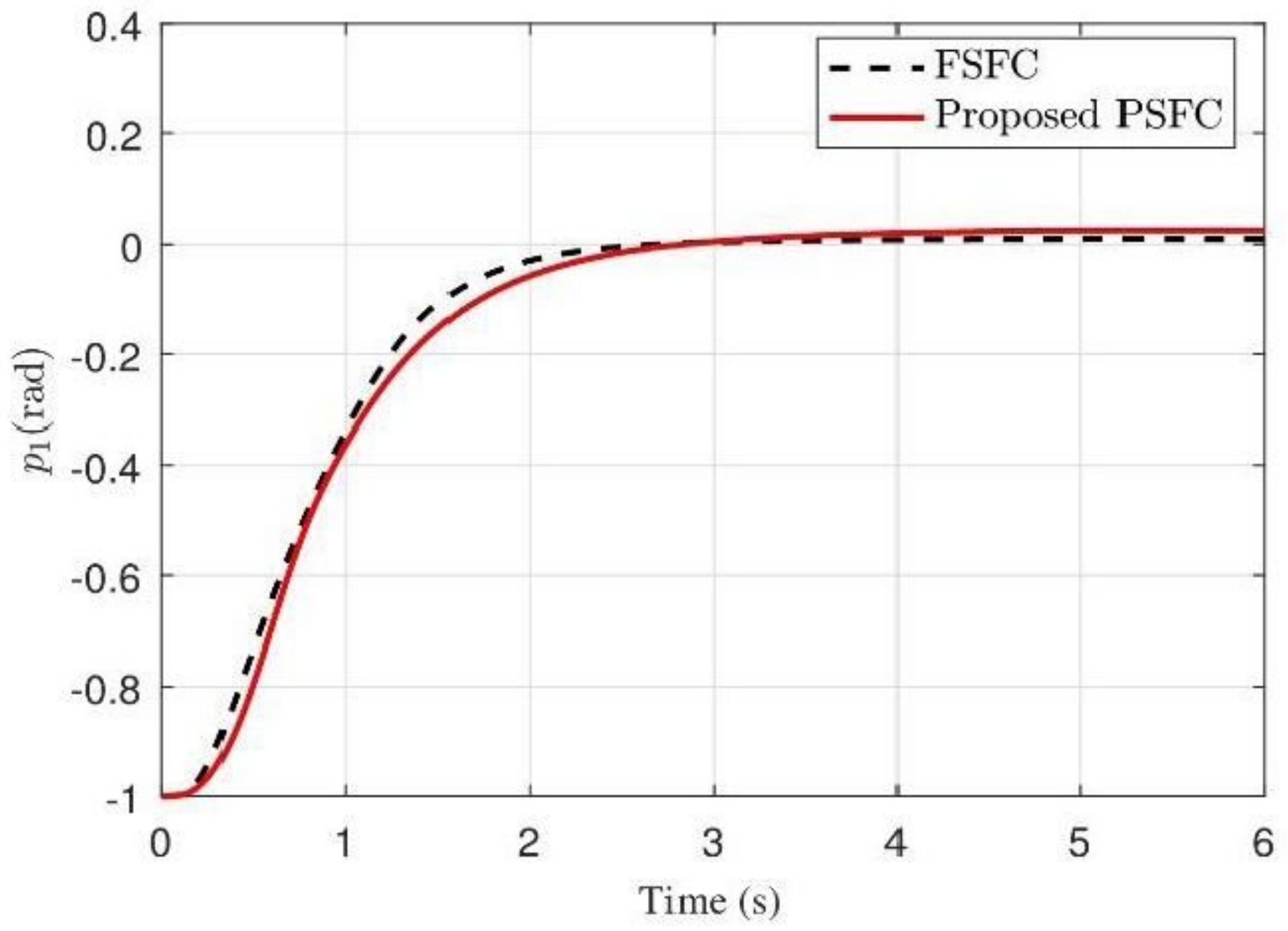

Figure 10

Tracking error $\mathrm{P} 1$ (case 2: qd = 1 rad) 


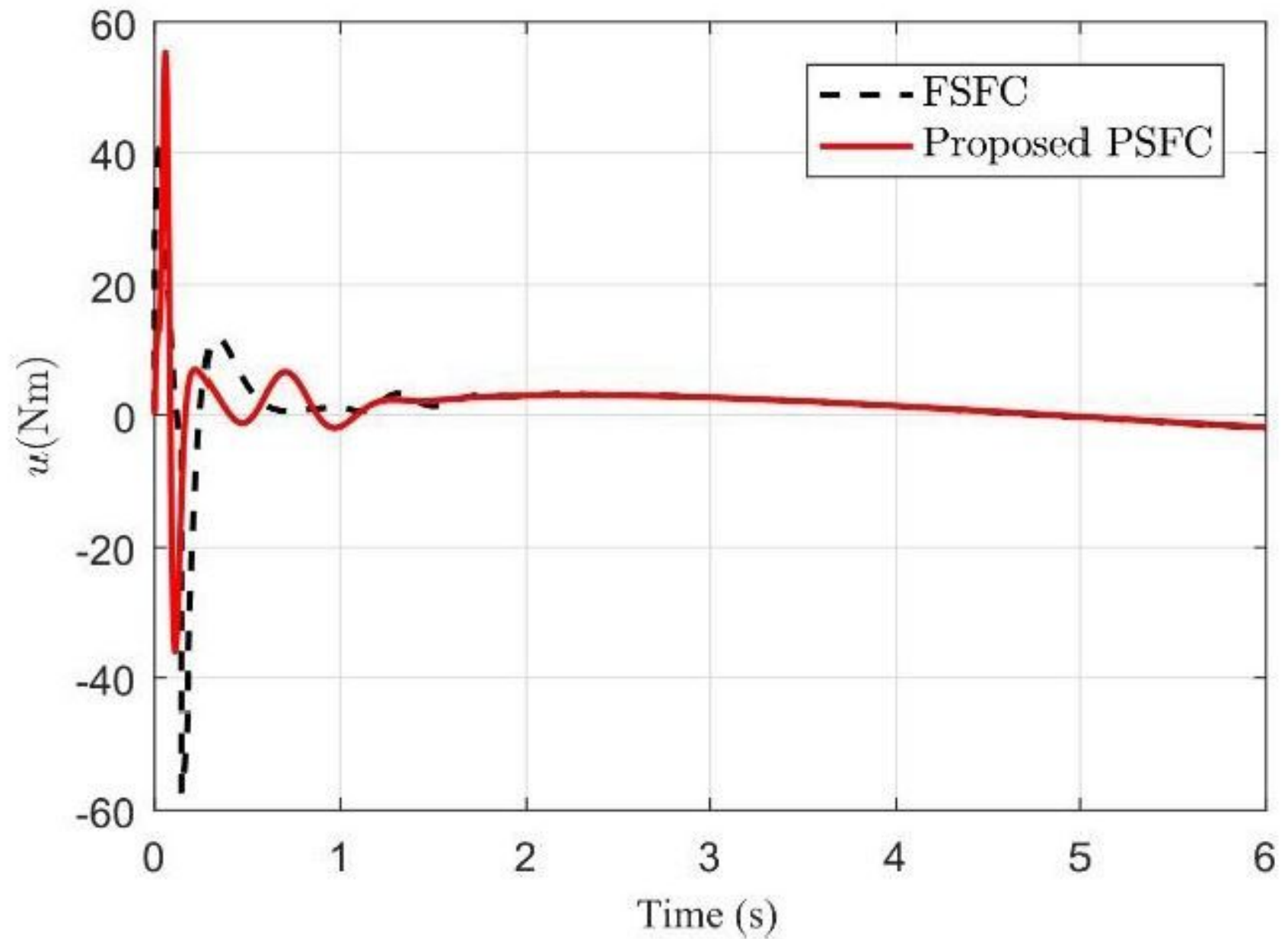

Figure 11

Control input $\mathrm{u}$ (case 2: $\mathrm{qd}=1 \mathrm{rad}$ ) 


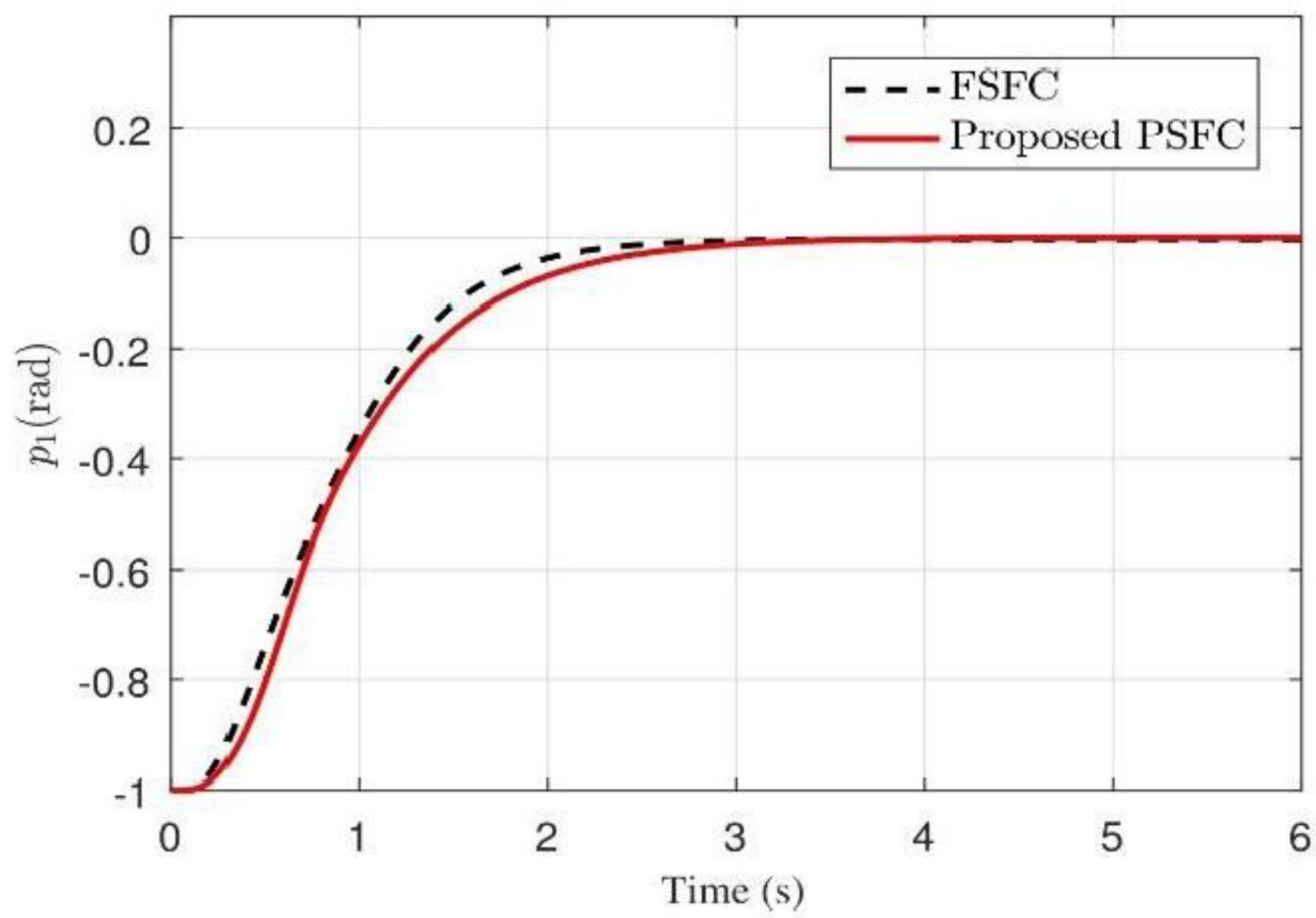

Figure 12

Tracking error $\mathrm{p} 1$ (case 2 : $q d=\cos (\mathrm{t})$ rad) 


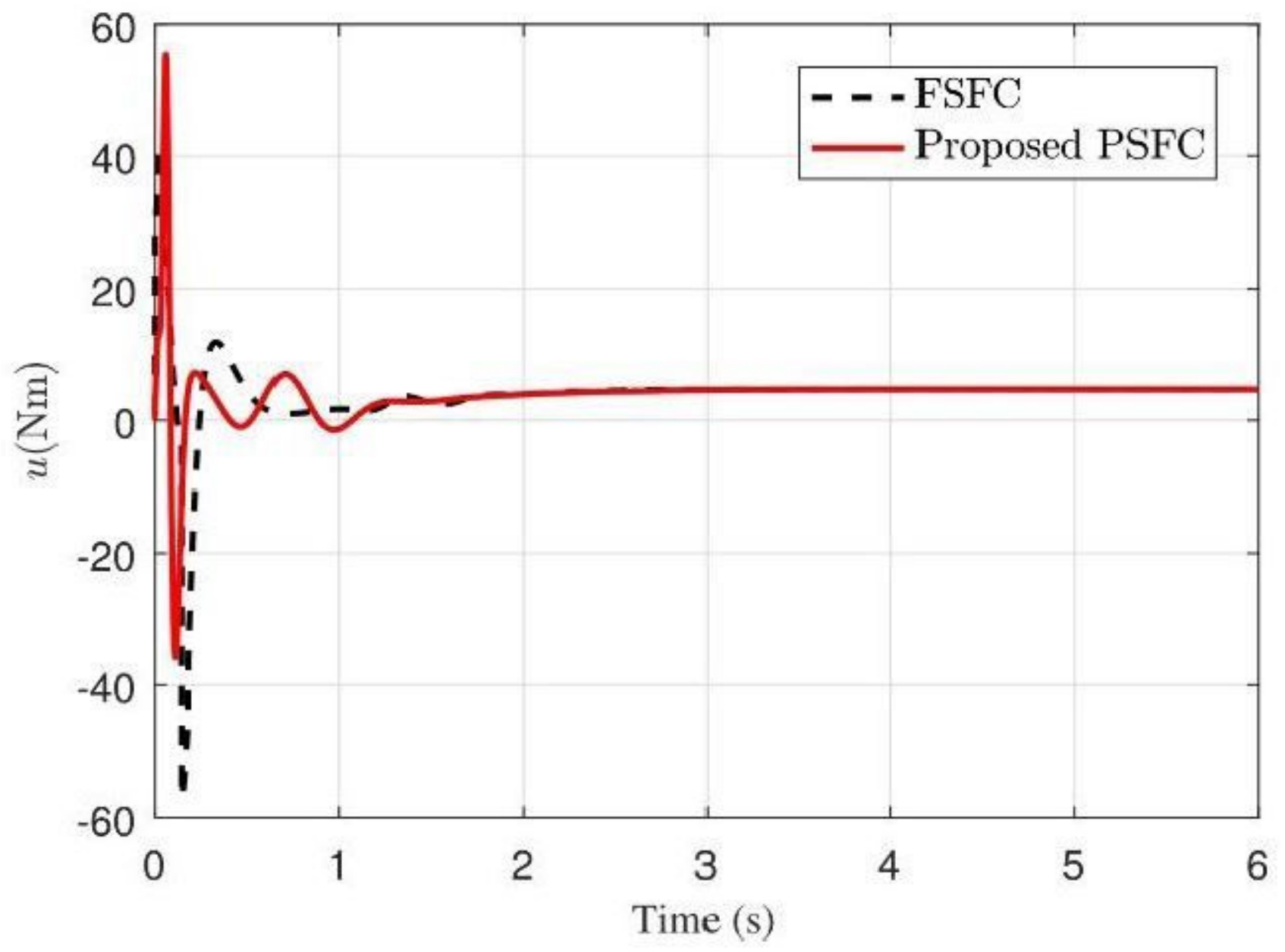

Figure 13

Control output u (case 2: $q d=\cos (t)$ rad) 


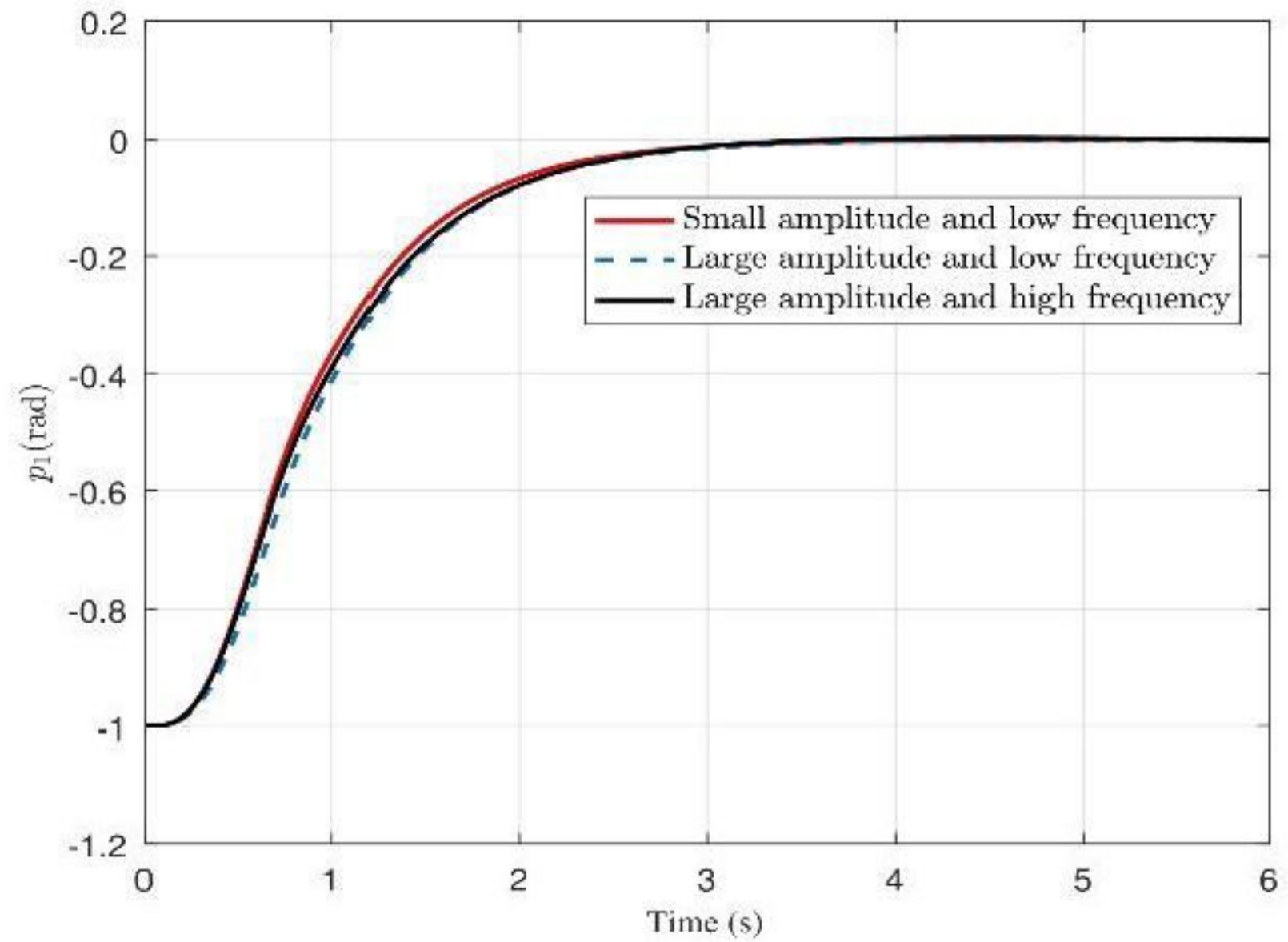

Figure 14

Tracking error P1 


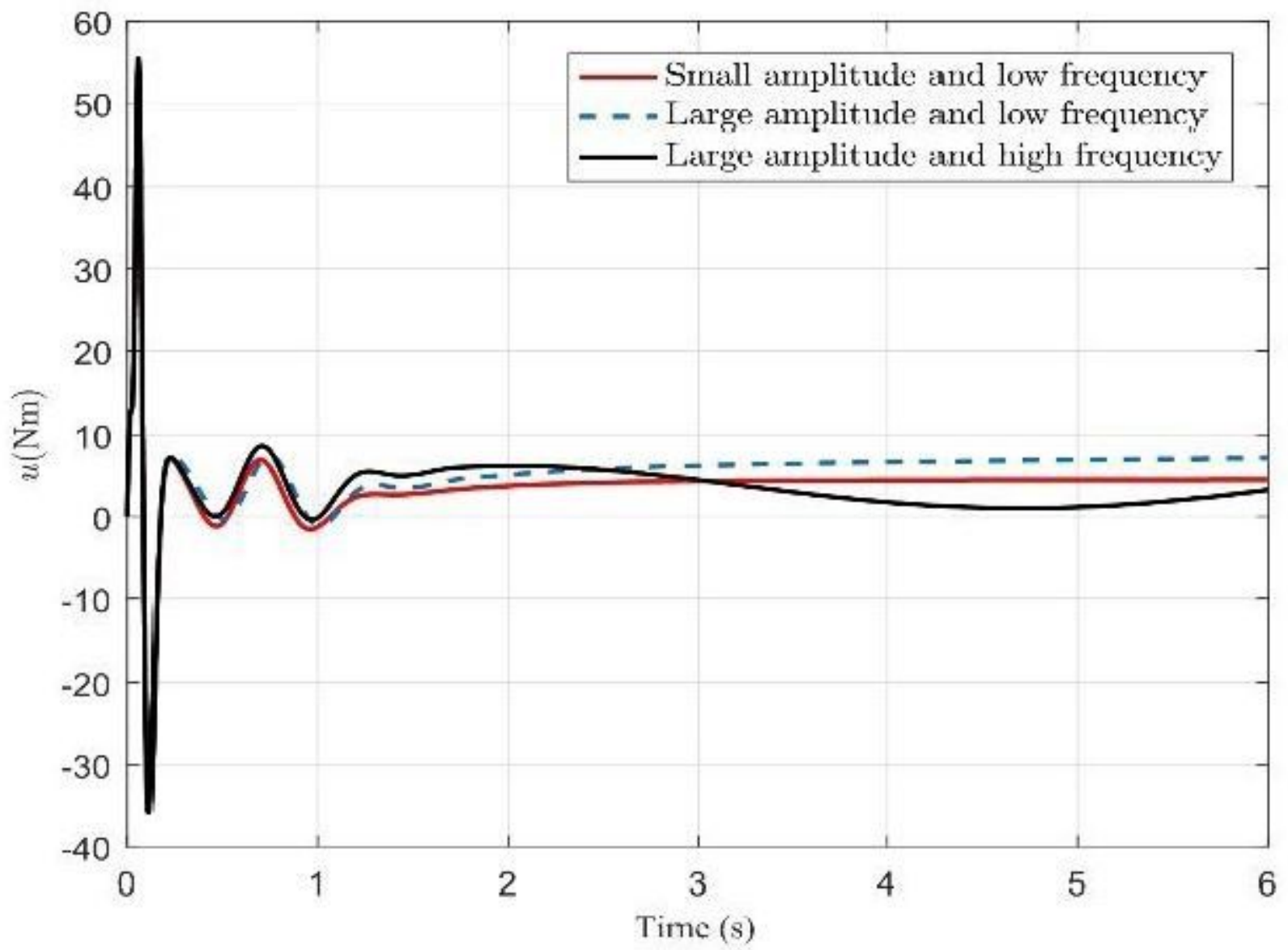

Figure 15

Control input u 